\title{
Medical Online Consultation Service in CAM at the University Hospital Zurich
}

Schmidt-Weitmann, Sabine ; Jenny, Katharina ; Neuhaus Bühler, Rachel ; Saller, Reinhard ; Brockes, Christiane

\begin{abstract}
Background: The University Hospital Zurich offers medical online consultation services in individual health concerns. We examined the need for users' information in the field of CAM. For this purpose, the content of the questions, the users' profiles, and the online responses of the physicians were analyzed. Methods: The retrospective study analyzed 154 (1.3\%) out of 11,827 questions and responses, selected by a literature-based keyword list between 2006 and 2007. They were evaluated by means of an inductive category system described by Mayring using a professional text analysis program (MAXQDA). Frequencies and mean values of the categories were statistically determined. Results: Users (aged $39.2 \pm 16$, females $61 \%$ ) asked questions, which were in $73 \%$ allocated to herbal medicine, $7 \%$ to homeopathy, and $2 \%$ to acupuncture. The questions referred to medical fields, such as gynecology (18\%), dermatology (13\%), psychiatry (11\%), and oncology $(8 \%)$. One third of the responses provided detailed information about herbal treatment options. Conclusions: The email-based online consultation service was used as a source of medical information in order to get more professional consultation in the field of CAM. Future scientific evaluation should investigate if online consultation services which are embedded in an environment of highly qualified health professionals may contribute to a better health literacy and empowerment of the patients.
\end{abstract}

DOI: https://doi.org/10.1159/000358502

Posted at the Zurich Open Repository and Archive, University of Zurich

ZORA URL: https://doi.org/10.5167/uzh-103149

Journal Article

Published Version

Originally published at:

Schmidt-Weitmann, Sabine; Jenny, Katharina; Neuhaus Bühler, Rachel; Saller, Reinhard; Brockes, Christiane (2014). Medical Online Consultation Service in CAM at the University Hospital Zurich. Forschende Komplementärmedizin, 21(1):19-24.

DOI: https://doi.org/10.1159/000358502 


\title{
Phytotherapie in der Geriatrie und Gerontologie
}

\author{
Reinhard Saller ${ }^{\mathrm{a}}$ Siegfried Kasper ${ }^{\mathrm{b}} \quad$ Wilfried Dimpfel $^{\mathrm{c}} \quad$ Egemen Savaskan $^{\mathrm{d}}$ Thomas Pfister ${ }^{\mathrm{e}}$ \\ Matthias Rostock ${ }^{\mathrm{f} g} \quad$ Claudia Witt $^{f}$ Christian Thuile $^{\mathrm{h}}$ Oliver Dasi Matthias Unger ${ }^{\mathrm{j}}$

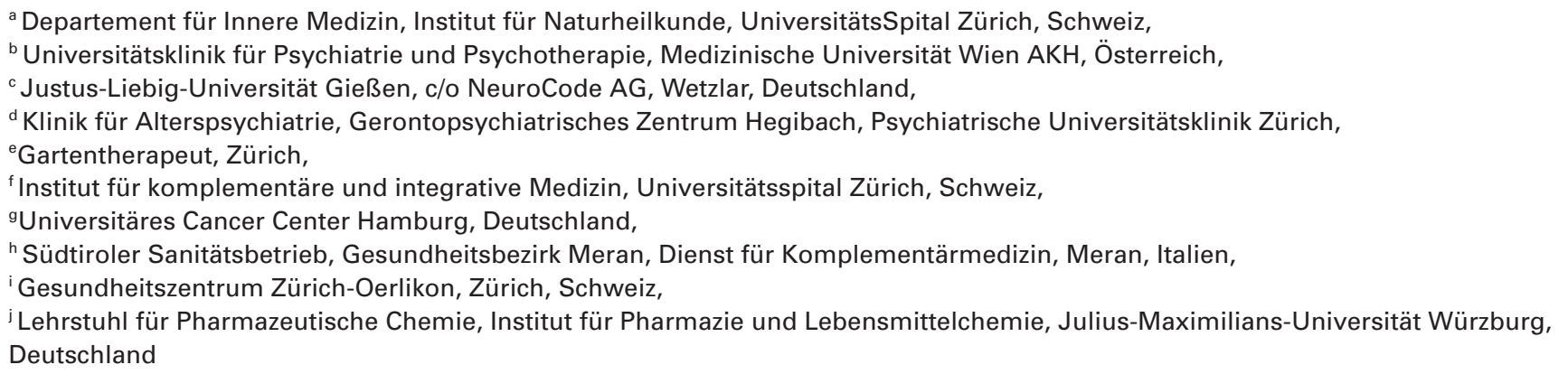

Der Charakter von Phytotherapeutika mit einem potentiell breiten Wirkungsspektrum (multi-target und multi-therapeutic) und in der Regel einer relativ geringen Häufigkeit von unerwünschten Wirkungen weist darauf hin, dass sie sich u.a. gerade auch in komplexen Therapiesituationen als bevorzugte Arzneimittel eignen könnten. Das höhere Lebensalter mit seinen oft multiplen Behandlungsanlässen und Alltagseinschränkungen sowie z.T. eigenen physiologischen und pathophysiologischen Abläufen ist ein solches Beispiel für häufige und schwierige Behandlungssituationen. Dieser Tagungsteil greift dies beispielsweise mit systematischen und narrativen Reviews zu ausgewählten Phytotherapeutika und deren Potential auf, ebenso mit Abhandlungen zur Behandlung des altersabhängig häufigen Prostatakarzinoms und den therapeutisch relevanten Problemen der alternden Haut sowie der Präsentation eines möglichst stilbildenden komplementärmedizinischen Pilotprojek- tes aus der Versorgungsforschung. Mit der Gartentherapie wird ein attraktives Behandlungsfeld aufgegriffen, in dem auch phytotherapeutische Gesichtspunkte eine bedeutsame Rolle spielen können.

Häufiger als in anderen Lebensabschnitten spielen im Alter Polypharmakotherapie und die Problematik eventueller Interaktionen eine Rolle. Die Einnahme von Phytotherapeutika wird dabei häufig und erstaunlich undifferenziert als zusätzliche Bedrohung propagiert. Eine kritische wie sachkundige Abhandlung zur Rolle von Phytotherapeutika im Zusammenhang mit Interaktionen vermag eine Reihe von pflanzlichen Arzneimitteln, die bei alten Menschen therapeutisch bedeutsam sind, als Pharmaka zu charakterisieren, deren Anwendung durch multimorbide Patienten aufgrund wissenschaftlicher Daten durchaus gerechtfertigt erscheint.

Reinhard Saller 


\section{Pflanzliche Antidepressiva, Anxiolytika und Antidementiva beim älteren Patienten}

\author{
Autor: Siegfried Kasper
}

\section{Weshalb sind Phytotherapeutika bei Älteren von besonderer Relevanz?}

Der aktuelle Demografiebericht der deutschen Bundesregierung geht davon aus, dass der Bevölkerungsanteil der mindestens 65-Jährigen von rund 20\% im Jahr 2011 bis 2016 auf $34 \%$ zunehmen wird [1]. Dank verbesserter gesundheitlicher Aufklärungsmaßnahmen, medizinischer Prophylaxe und therapeutischer Möglichkeiten ist auch in den anderen westlichen Industrienationen ein vergleichbarer Anstieg der Lebenserwartung und eine Zunahme des Bevölkerungsanteils älterer Menschen zu beobachten. Somit stellen für die Medizin nicht nur die typischen «Alterskrankheiten» eine zunehmende Herausforderung dar, sondern auch die adäquate Versorgung eines immer älter werdenden, oft multimorbiden Patientenkollektivs allgemein.

Die durch die Multimorbidität vieler älterer Patienten bedingte Polypharmakotherapie verursacht besonders im ambulanten Bereich oft unabsehbare Risiken. Im Unterschied zu synthetischen Arzneimitteln sind Phytopharmaka komplexe Vielstoffgemische mit einem vergleichsweise breiten Wirkprofil. Wirkmechanistisch bedingte Nebenwirkungen, insbesondere solche mit schwerer Ausprägung, sind zumeist selten oder sogar unbekannt; das Interaktionspotenzial ist für viele pflanzliche Präparate im Vergleich zu synthetischen Therapeutika gering. Bei nachgewiesener Wirksamkeit bieten sie sich daher gerade bei älteren Menschen mit oft chronischen Erkrankungen als gut verträgliche, die Lebensqualität der Betroffenen verbessernde Alternative zu synthetischen Therapeutika an.

In den nicht speziell geriatrischen Indikationen stehen generell nur wenige Studien mit Fokus auf ältere Patienten zur Verfügung. Selbst in klinischen Prüfungen ohne Altersbeschränkung nach oben sind Ältere im Vergleich zu ihrem Bevölkerungsanteil eher unterrepräsentiert, weil sie aufgrund von Begleiterkrankungen oder -medikation häufiger von der Studienteilnahme ausgeschlossen werden als Jüngere. Deswegen liegen für Arzneimittel häufig nur unzureichende Wirksamkeitsdaten aus geriatrischen Kollektiven vor. Das gilt auch für Studien mit Phytotherapeutika.

Für den Einsatz von Phytotherapeutika an älteren Menschen berichten wir beispielhaft über die Wirksamkeit der Behandlung von Angststörungen mit Lavendelöl, von Depressionen mit Hypericum-Extrakt sowie von Demenz-Patienten mit Ginkgo-biloba-Extrakt.

\section{Methodik}

Innerhalb der Indikationsgebiete Angststörungen und Demenz wurden für die geeigneten randomisierten, kontrollierten Phase-3-Studien mit den Studienteilnehmern im Altersbereich $\geq 60$ Jahre Subgruppenanalysen der Wirksamkeitshauptzielparameter durchgeführt und anschließend mittels Metaanalyse kombiniert. Soweit nicht anders beschrieben, basieren die Analysen auf den bei der konfirmatorischen Wirksamkeitsauswertung der Primärstudien herangezogenen Kollektiven (Full Analysis Sets). Die Behandlungsgruppenvergleiche innerhalb der Studien erfolgten mittels Kovarianzanalysen (mit Behandlung als Faktor und dem Baseline-Wert als Kovariable) oder Varianzanalysen. Da in der Indikation Depression von den 4 geeigneten Studien 2 placebokontrolliert waren, wurden die Wirkungen bei älteren Patienten und jüngeren Patienten in dem gepoolten Datensatz der 4 Studien verglichen. Zusätzlich wurde die Wirkung in der gepoolten Placebogruppe beschrieben. Zweiseitige $p$-Werte $\leq 0,05$ werden als deskriptiv signifikant angesehen.

\section{Lavendelöl-Zubereitung Silexan bei Angststörungen}

Silexan, der Wirkstoff des Produkts Lasea ${ }^{\circledR}$ (Dr. Willmar Schwabe GmbH \& Co. KG, Karlsruhe), ist ein patentierter Wirkstoff, der ein durch Dampfdestillation aus Blüten von Lavandula angustifolia gewonnenes, ätherisches Öl enthält und in allen Qualitätsparametern den Vorgaben der Monographie Lavendelöl aus der Ph. Eur. entspricht. Es übertrifft die Qualitätsbeschreibung der Monographie bei den für Wirksamkeit und Verträglichkeit relevanten Inhaltsstoffen. Zur Metaanalyse standen 4 placebokontrollierte Studien mit Patienten zur Verfügung, die an einer generalisierten Angsterkrankung [2; Möller et al., in Vorbereitung], an einer subsyndromalen Angststörung [3] oder an angstbezogenen Unruhezuständen und Schlafstörungen [4] litten. Insgesamt 112 der Studienteilnehmer waren mindestens 60 Jahre alt (Mittel: 63 Jahre); $78 \%$ von ihnen waren Frauen. Diese im Rahmen der Metaanalyse ausgewerteten Patienten erhielten $1 \times 80 \mathrm{mg} / \mathrm{Tag}$ Silexan $(\mathrm{n}=55)$ oder Placebo $(\mathrm{n}=57)$ über einen Therapiezeitraum von 10 Wochen. Ausgewertet wurden die Veränderung des Gesamtscores der Hamilton Angstskala (HAMA) [5] sowie des mit der Clinical Global Impressions-Skala (CGI) [6] erfassten, psychiatrischen Gesamtbilds.

In der Subgruppe der $\geq 60$-Jährigen nahm der HAMA-Gesamtscore in 3 der 4 Primärstudien im Behandlungsverlauf unter Silexan deutlich stärker ab als unter Placebo. Im gepoolten Datensatz aller Studien ging der Score unter Silexan von 25,1 $\pm 5,7$ (Mittel \pm Standardabweichung) Punkten bei Behandlungsbeginn auf 11,6 \pm 7,4 Punkte am Behandlungsende zurück, verglichen mit einem Rückgang von 25,2 \pm 5,8 Punkte auf 15,7 \pm 7,6 Punkte unter Placebo. In der Metaanalyse ergab sich eine statistisch signifikante Differenz der varianzanaly- 
tisch adjustierten Mittelwerte von 4,3 Punkten zugunsten von Silexan $(\mathrm{p}<0,01)$.

Im Gesamtbild der über 60-Jährigen lag der mittlere Schweregrad der Erkrankung (CGI Item 1) vor Behandlungsbeginn in allen Primärstudien zwischen 4 («mäßig krank») und 5 Punkten («deutlich krank»), mit Mittelwerten von jeweils 4,5 \pm 0,6 Punkten für Silexan und Placebo im gepoolten Datensatz. In 3 der 4 Primärstudien zeigte sich unter Silexan im Therapieverlauf eine deutlich stärkere Verbesserung des Globalurteils als unter Placebo, wobei die Mittelwerte im gepoolten Datensatz bei den mit Silexan behandelten Patienten auf 3,0 \pm 1,1 Punkte (3 Punkte: «leicht krank») und in der Placebogruppe auf 3,4 \pm 1,3 Punkte abnahmen. In der Metaanalyse ergab sich für die Score-Abnahme eine Gruppendifferenz der adjustierten Mittelwerte von 0,3 Punkten. Bei der Veränderung des klinischen Gesamtbilds (CGI Item 2) ergaben sich am Behandlungsende im gepoolten Datensatz Mittelwerte von 2,1 $\pm 1,0$ Punkten für Silexan und von 2,8 \pm 1,3 Punkten für Placebo (2 Punkte: «viel besser»; 3 Punkte: «nur wenig besser»). Der Gruppenunterschied von 0,6 Punkten war in der Metaanalyse zugunsten von Silexan signifikant $(p<0,01)$.

Die Ergebnisse belegen in der Altersgruppe der mindestens 60-Jährigen signifikante Vorteile von Silexan gegenüber Placebo sowohl hinsichtlich der Anxiolyse als auch im psychiatrischen Gesamtbild.

\section{Johanniskrautextrakt WS ${ }^{\circledR} 5570$ bei depressiven Erkrankungen}

WS $^{\circledR}$ 5570, der Wirkstoff des Produkts Neuroplant ${ }^{\circledR}$ (Dr. Wilmar Schwabe GmbH \& Co. KG, Karlsruhe), ist ein stabilisierter Trockenextrakt aus Johanniskraut (3-7:1), Auszugsmittel Methanol 80\% (V/V), mit einem definierten Gehalt von 3-6\% Hyperforin, 0,1-0,3\% Hypericin, mindestens 6\% Flavonoide und mindestens $1,5 \%$ Rutin. Die in die Metaanalyse einbezogenen Daten entstammen 4 klinischen Prüfungen, wobei jeweils die 6-wöchige Akutphase der Behandlung ausgewertet wurde: Zwei der Studien verglichen bei Patienten mit leichten bis mittelschweren Depressionen $900 \mathrm{mg} / \mathrm{Tag} \mathrm{WS}^{\circledR}$ 5570 [7] bzw. 600 und 1200 mg/Tag WS ${ }^{\circledR} 5570$ [8] mit Placebo. In der 3. Studie erhielten mittelschwer bis schwer depressive Patienten 900 oder 1800 mg/Tag WS ${ }^{\circledR} 5570$ bzw. 20 oder 40 mg/ Tag Paroxetin [9]. Beim 4. Projekt handelte es sich um eine Langzeitstudie mit Patienten, die an leichten bis mittelschweren rekurrenten depressiven Episoden litten [10]. Die Teilnehmer durchliefen vor der Langzeitphase eine 6-wöchige, einfachblinde Akutphase mit Einnahme von $900 \mathrm{mg} / \mathrm{Tag} \mathrm{WS}^{\circledR}$ 5570, die zur gepoolten Auswertung herangezogen wurde.

116 der im Rahmen der 4 Studien mit WS ${ }^{\circledR} 5570$ behandelten Patienten waren mindestens 60 Jahre alt. Als Vergleichsgruppen dienten die unter 60 -jährigen, ebenfalls mit WS ${ }^{\circledR} 5570$ behandelten Patienten ( $\mathrm{n}=942$; Mittel: 44 Jahre) sowie die Patienten der Placebogruppen der ersten beiden Studien
( $\mathrm{n}=270$; Mittel: 43 Jahre; 16 Patienten $\geq 60$ Jahre). Zwischen $70 \%$ und $78 \%$ der Patienten in den Gruppen waren weiblich.

Die Schwere der Depression wurde in allen Studien primär mithilfe der Hamilton Depressionsskala (HAMD) [11] beurteilt. Vor Behandlungsbeginn lagen die mittleren HAMD-Gesamtscores aller verglichenen Gruppen im gepoolten Datensatz aus allen Primärstudien homogen im Bereich zwischen 22,4 $\pm 2,8$ und 23,5 $\pm 3,1$ Punkten. Zwischen der Baseline und dem Ende der 6-wöchigen Akutphase reduzierte sich der Score bei den $\geq 60$-jährigen, mit WS ${ }^{\circledR} 5570$ behandelten Patienten um 13,2 \pm 6,0 Punkte, bei den mit WS ${ }^{\circledR} 5570$ behandelten Patienten $<60$ Jahre um 12,3 \pm 6,6 Punkte und bei den Patienten der Placebogruppen um 7,5 \pm 7,4 Punkte. Es gab keinen statistisch signifikanten Unterschied zwischen der HAMD-Abnahme bei den älteren und jüngeren Patienten.

Patienten mit einer HAMD-Gesamtscore-Abnahme von $\geq 50 \%$ des Ausgangswerts unter Akutbehandlung wurden als Responder klassifiziert, solche mit einem Endwert $\leq 7$ Punkte als Remitter. Die Responder- und Remitter-Raten lagen bei den $\geq 60$-jährigen, $\mathrm{WS}^{\circledR} 5570$ behandelten Patienten bei $75,9 \%$ und $27,6 \%$, bei den jüngeren Hypericum-Patienten bei 70,9\% und 31,9\% und unter Placebo bei 39,3\% und bei 19,3\%.

Insgesamt zeigen die Ergebnisse für WS ${ }^{\circledR} 5570$ bei den $\geq 60$-Jährigen einen ebenso ausgeprägten antidepressiven Effekt wie bei jüngeren Patienten. In beiden Altersgruppen war die Wirksamkeit des Johanniskrautextrakts derjenigen von Placebo deutlich überlegen.

\section{Ginkgo-biloba-Extrakt EGb $761^{\circledR}$ bei Demenz-Patienten}

Bei EGb $761^{\circledR}$, dem Wirkstoff des Produkts Tebonin ${ }^{\circledR}$ (Dr. Wilmar Schwabe GmbH \& Co. KG, Karlsruhe), handelt es sich um einen Trockenextrakt aus Ginkgo-biloba-Blättern (DEV 35-67: 1), Auszugsmittel Aceton 60\% (m/m). Der Extrakt ist quantifiziert auf 22-27\% Ginkgoflavonglykoside, sowie 5-7\% Terpenlactone, davon 2,8-3,4\% GinKgolide A, B und C und 2,6-3,2\% Bilobalid und enthält unter 5 ppm Ginkgolsäuren. Die vorgestellte Metaanalyse basiert auf 4 klinischen Prüfungen, deren Teilnehmer an Alzheimer-Demenz, vaskulärer Demenz oder einer Mischform mit neuropsychiatrischen Symptomen erkrankt waren [12-15]. Die Patienten erhielten 240 mg/Tag EGb $761^{\circledR}$ oder Placebo über einen Behandlungszeitraum von 22 oder 24 Wochen.

In die Primärstudien wurden Patienten ab einem Alter von 50 Jahren eingeschlossen; jedoch befand sich die Mehrzahl der Teilnehmer in dem in die Metaanalyse einbezogenen Altersbereich $\geq 60$ Jahre, so dass insgesamt 1108 Patienten ausgewertet werden konnten (EGb $761^{\circledR}: 571$; Placebo: 537). Im ausgewerteten Kollektiv lagen die Altersmittel der Studien zwischen 69 und 72 Jahren; zwischen $57 \%$ und $71 \%$ der Studienteilnehmer waren Frauen.

Die demenzbedingten psychopathologischen Störungen sowie deren Auswirkungen auf die Betreuer der Patienten wur- 
den bei allen Projekten mithilfe des Neuropsychiatrischen Inventars (NPI) [16] erfasst. Die Metaanalyse der Veränderung des NPI zwischen Baseline und Behandlungsende zeigte signifikante Vorteile für EGb $761^{\circledR}$ im Vergleich zu Placebo, mit Mittelwertdifferenzen von 3,1 Punkten im Symptom-Gesamtscore $(\mathrm{p}<0,001)$ und von 1,8 Punkten im Caregiver Distress Score $(\mathrm{p}<0,001)$. Vorteile für EGb $761^{\circledR}$ ergaben sich auch in den Metaanalysen für alle 12 Einzelsymptome des NPI und für 11 der 12 Items beim Caregiver Distress, in beiden Fällen mit signifikanten Unterschieden bei Agitiertheit, Depression, Angst, Apathie, Reizbarkeit, motorisches Verhalten und Schlaf (jeweils $\mathrm{p}<0,05$ ).

In der Indikation Demenz besteht die Zielpopulation von Ginkgo-Präparaten naturgemäß überwiegend aus älteren $\mathrm{Pa}-$ tienten. Die durchgeführten Metaanalysen belegen die Wirksamkeit von EGb $761^{\circledR}$ bei Demenz im kognitiven Bereich sowie zur Reduktion demenzassoziierter psychopathologischer Störungen in der Hauptzielpopulation der $\geq 60$-Jährigen. Die beobachteten Vorteile für die Patienten gingen mit einer ebenso deutlich ausgeprägten Reduktion der Belastung für deren Betreuer einher.

\section{Fazit}

Die in der Subgruppe der über 60-Jährigen durchgeführten Metaanalysen spiegeln im Wesentlichen die Ergebnisse der Gesamtkollektive der analysierten Studien wider. Die untersuchten Wirkstoffe Silexan und WS ${ }^{\circledR} 5570$ waren in ihrer jeweiligen Indikation bei älteren Patienten ebenso wirksam wie in nichtgeriatrischen Patientenkollektiven und eignen sich somit auch für die Behandlung älterer Menschen. Die Wirksamkeit und Verträglichkeit von EGb $761^{\circledR}$ wurden ohnehin in einem geriatrischen Kollektiv belegt.

\section{Literatur}

1 Bundesministerium des Innern: Demografiebericht. Bericht der Bundesregierung zur demografischen Lage und künftigen Entwicklung des Landes. Berlin: Bundesministerium des Innern, 2011.

2 Kasper S, Gastpar M, Müller WE, Volz H-P, Möller H-J, Schläfke S, Dienel A: Lavender oil preparation Silexan is effective in generalized anxiety disorder - a randomized, double-blind comparison to placebo and paroxetine. Int J Neuropsychopharmacol 2014;17:859-869.

3 Kasper S, Gastpar M, Müller WE, Volz HP, Möller HJ, Dienel A, Schläfke S: Silexan, an orally administered Lavandula oil preparation, is effective in the treatment of 'subsyndromal' anxiety disorder: a randomized, double-blind, placebo controlled trial. Int Clin Psychopharmacol 2010;25:277-287.

4 Kasper S, Anghelescu I, Dienel A: Efficacy of Silexan (WS $\left.{ }^{\circledR} 1265\right)$ in Patients with Restlessness and Sleep Disturbance. Annual Congress of the German Society for Psychiatry and Psychotherapy (DGPPN). Berlin, Germany, 2010.

5 Hamilton M: Hamilton Anxiety Scale (HAMA); in Guy W (ed): ECDEU Assessment Manual for Psychopharmacology. Rockville, MD, U.S. National Institute of Health, Psychopharmacology Research Branch, 1976, pp 193-197.

6 National Institute of Mental Health: 12 - CGI. Clinical Global Impressions; in Guy W (ed): EDCEU Assessment in Psychopharmacology. Rockville, MD, U.S National Institute of Mental Health, Psychopharmacology Research Branch, 1970, pp 217-222.
7 Lecrubier Y, Clerc G, Didi R, Kieser M: Efficacy of St. John's wort extract WS 5570 in major depression: a double-blind, placebo-controlled trial. Am J Psychiatry 2002;159:1361-1366.

8 Kasper S, Anghelescu I, Szegedi A, Dienel A, Kieser M: Superior efficacy of St John's wort extract WS ${ }^{\circledR} 5570$ compared to placebo in patients with major depression: a randomized, double-blind, placebo-controlled, multi-center trial. BMC Med 2006;4:14.

9 Szegedi A, Kohnen R, Dienel A, Kieser M: Acute treatment of moderate to severe depression with hypericum extract WS 5570 (St John's wort): randomised controlled double blind non-inferiority trial versus paroxetine. BMJ 2005;330:503-506.

10 Dienel A, Kasper S, Kieser M: Hypericum extract WS ${ }^{\circledR} 5570$ has a beneficial effect in continuation treatment of recurrent depression. Pharmacopsychiatry 2005;38:236

11 Hamilton M: Development of a rating scale for primary depressive illness. Br J Soc Clin Psychol 1967;6:278-296.

12 Napryeyenko O, Borzenko I, GINDEM-NP Study Group: Ginkgo biloba special extract in dementia with neuropsychiatric features. A randomised, placebo-controlled, double-blind clinical trial. Arzneimittelforschung 2007;57:4-11.

13 Nikolova G, Yancheva S, Raychev I, Hoerr R, PLAGIN Study Group: Ginkgo biloba extract in dementia: a 22-week randomised, placebo-controlled, double-blind trial. Bulgarian Neurol 2013;14:139-143.

14 Herrschaft H, Nacu A, Likhachev S, Sholomov I, Hoerr R, Schlaefke S: Ginkgo biloba extract EGb 761(R) in dementia with neuropsychiatric features: a randomised, placebo-controlled trial to confirm the efficacy and safety of a daily dose of 240 mg. J Psychiatr Res 2012;46:716-723.

15 Ihl R, Bachinskaya N, Korczyn AD, Vakhapova V, Tribanek M, Hoerr R, Napryeyenko O, Gotaday Study Group: Efficacy and safety of a once-daily formulation of Ginkgo biloba extract EGb 761 in dementia with neuropsychiatric features: a randomized controlled trial. Int J Geriatr Psychiatry 2011;26:1186-1194.

16 Cummings JL:The Neuropsychiatric Inventory: assessing psychopathology in dementia patients. Neurology 1997;48:S10-16.

\section{Mit pflanzlichen Arzneimitteln erzeugte Signale im EEG und deren Bedeutung für die Klinik in der Geriatrie}

\section{Autor: Wilfried Dimpfel}

Die elektrische Hirnaktivität lässt sich heutzutage in Form des Elektroenzephalogramms (EEG) relativ einfach ohne großen Aufwand mithilfe einer Elektrodenkappe, einem Verstärker sowie einem Computer messen. Das aus einer solchen Erfassung der elektrischen Aktivität resultierende Rohsignal im Bereich von Millionstel Volt lässt sich jedoch kaum in Richtung einer Medikamentenwirkung direkt interpretieren. Abhilfe schafft eine Frequenzanalyse des Signals mittels einer Fast Fourier Transformation. Das Ergebnis ist ein Leistungsdichtespektrum, welches in mehrere Frequenzbereiche unterteilt wird. Wir sprechen von Delta-, Theta-, Alpha- und Beta-Wellen. Sie reflektieren u.a. Änderungen der chemischen Neurotransmitteraktivität, wie wir heute wissen. Da Medikamente - auch die pflanzlichen Präparate - auf dieser chemischen Ebene angreifen, sind Änderungen der elektrischen Aktivität zu erwarten und wurden auch als Elektropharmakogramm beschrieben. Der Frequenzgehalt des EEGs ist bei Männern und Frauen verschieden und ändert sich auch im Laufe des Lebens, erkennbar an einer signifikanten Zunahme der schnellen Beta-Wellen. Daher reagieren ältere Menschen unter Umständen anders auf Medikamente, die in diesem Bereich der glutamatergen und GABAergen Transmission angreifen, wie zum Beispiel bei der Einnahme von Diazepam. Mit zunehmendem Alter kommt es bei vielen Menschen vor- 


\section{d2-Test/Augen auf}

\section{HC $n=44$}

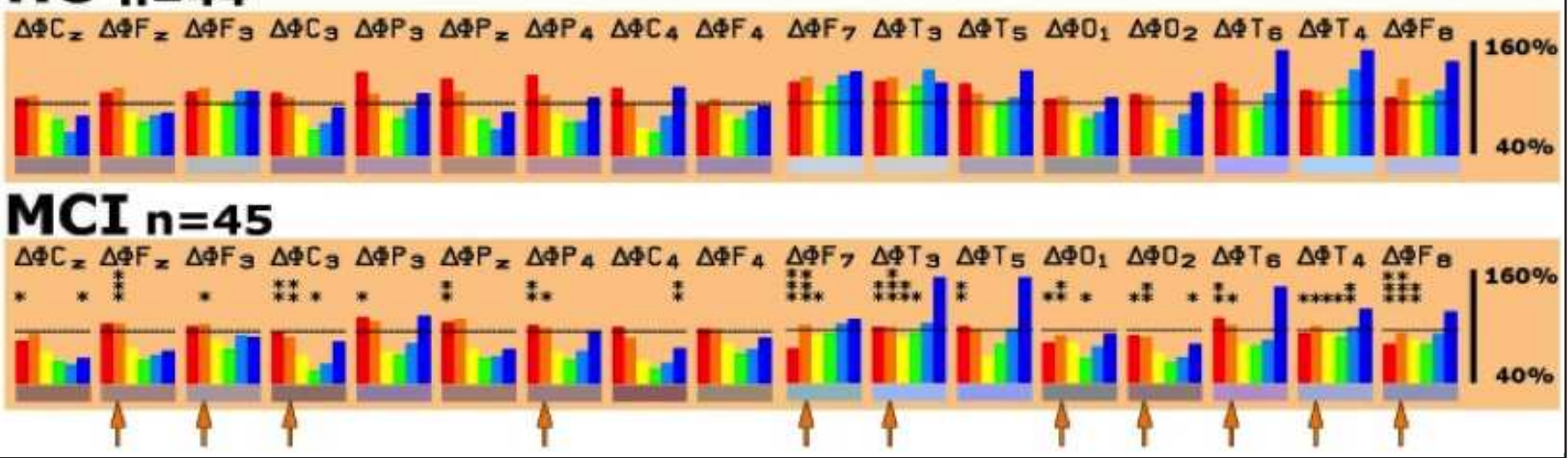

Abb. 1. Vergleich gesunder (HC) mit kognitiv beeinträchtigten (MCI) Gehirnen bezüglich der elektrischen Leistung in 6 verschiedenen Frequenzbereichen (farblich als Säulen dargestellt) an 17 Elektrodenpositionen $(\mathrm{C}=$ central, $\mathrm{F}=$ frontal, $\mathrm{P}=$ parietal, $\mathrm{T}=$ temporal, $\mathrm{O}=$ occipital) während der Durchführung des d2-Konzentrationstestes. Man beachte die deutlich geringer ausgeprägte in oranger Farbe dargestellte Theta-Aktivität bei kognitiver Beeinträchtigung (MCI) (s. auch orangene Pfeile bei niedriger statistischer Irrtumswahrscheinlichkeit).

wiegend zu zwei Phänomenen: 1. zu einem Nachlassen kognitiver Leistungen (engl.: mild cognitive impairment (MCI)), welche im Rahmen der Durchführung eines kurzen Tests (DemTect) erfasst wird, und 2. zu Schlafstörungen, die anamnestisch erfasst werden. Beide Beeinträchtigungen der Lebensqualität möchte man medikamentös behandeln. Die Voraussetzung hierfür ist jedoch die quantitative Erfassbarkeit, die die Auswertung des EEG in besonderem Maße bietet. Betrachten wir zunächst die mentale Leistungsfähigkeit. Hier zeigt ein Vergleich von gesunden Gehirnen mit denen derjenigen Menschen, die über leichte Vergesslichkeit klagen (DemTec: Score 8-12), dass statistisch signifikant weniger Theta-Aktivität während der Durchführung eines Konzentrationstestes vorliegt. Abbildung 1 dokumentiert dies in deutlicher Form:

Bei der Durchführung eines Gedächtnistestes sind es die Alpha2-Wellen, die bei leichter kognitiver Beeinträchtigung deutlich geringer ausgeprägt sind. Daraus folgt, dass man nach Medikamenten suchen muss, die Theta- und Alpha2-Wellen beeinflussen können, um diesen Menschen zu helfen.

Die zweite wichtige Beeinträchtigung der Lebensqualität sind Schlafstörungen. Auch diese lassen sich anhand der Ableitung des EEG während der Schlafphase quantitativ erfassen. Damit lässt sich auch eine erfolgreiche Therapie überprüfen. Abbildung 2 zeigt ein repräsentatives Beispiel anhand einer innovativen Form der Schlafdokumentation, in der das Verhältnis der Theta-, Beta1- und Beta2-Wellen zueinander für den Verlauf der Nacht dargestellt ist (Beginn = links oben). Neben der klassischen Darstellung der Schlaftiefe anhand des von mir entwickelten patentierten Schlafindexes (Spektraler Frequenzindex: «SFx») erlaubt diese Form der Dokumentation der Schlafqualität auch die Darstellung einer Medikamentenwirkung wie am Beispiel eines Schweizer Baldrian-Hopfen-Präparates in Abbildung 2 gezeigt werden kann:

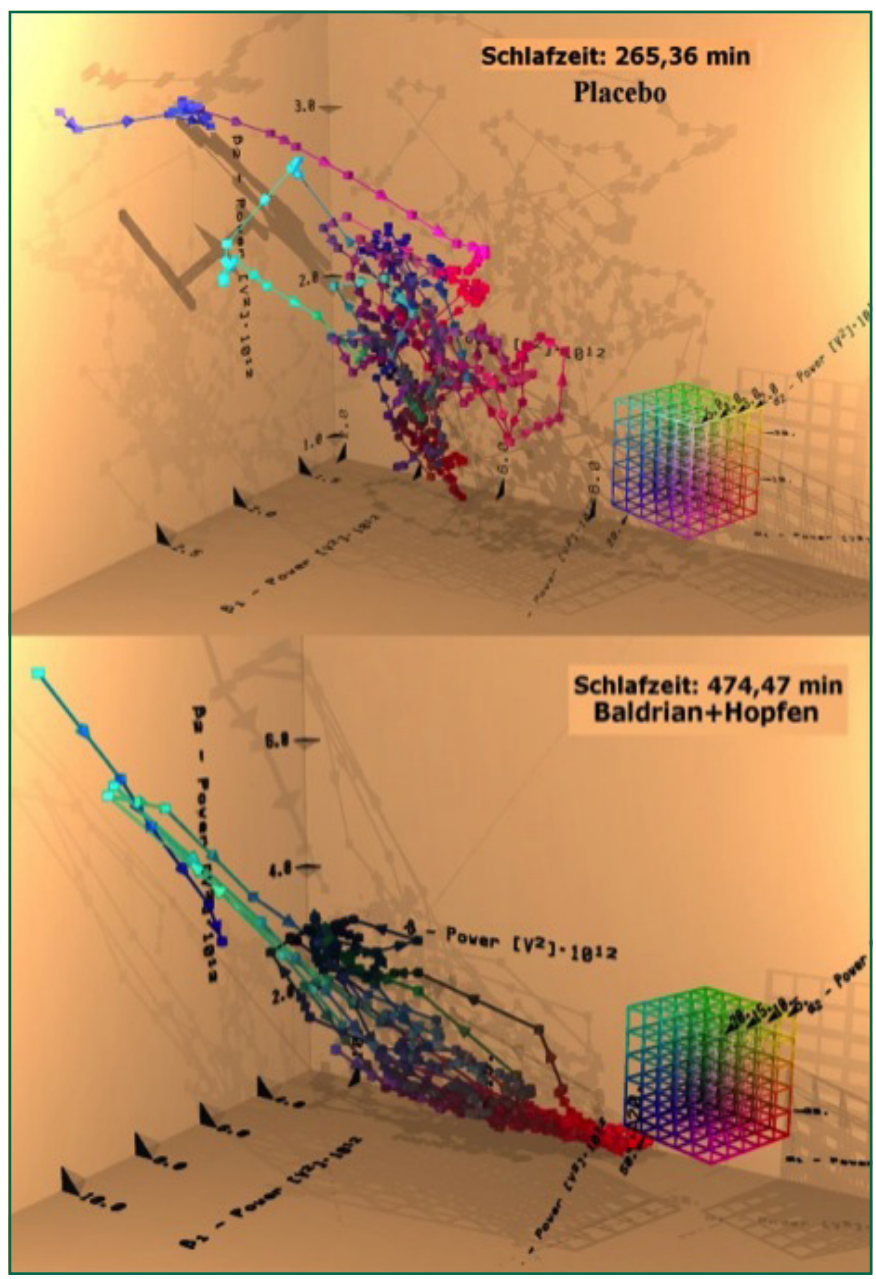

Abb. 2. Verlauf der Theta-, Beta1- und Beta2-Wellen (x-, y- und z-Achse) als «Schlafspur» über die Nacht unter Placebo-Gabe (oben) bzw. nach Einnahme eines Baldrian-Hopfen-Präparates. Die drei übrigen Frequenzen wurden mithilfe des RGB-Verfahrens (wie im TV) als additive Farbmischung kodiert (s. Farbwürfel). 


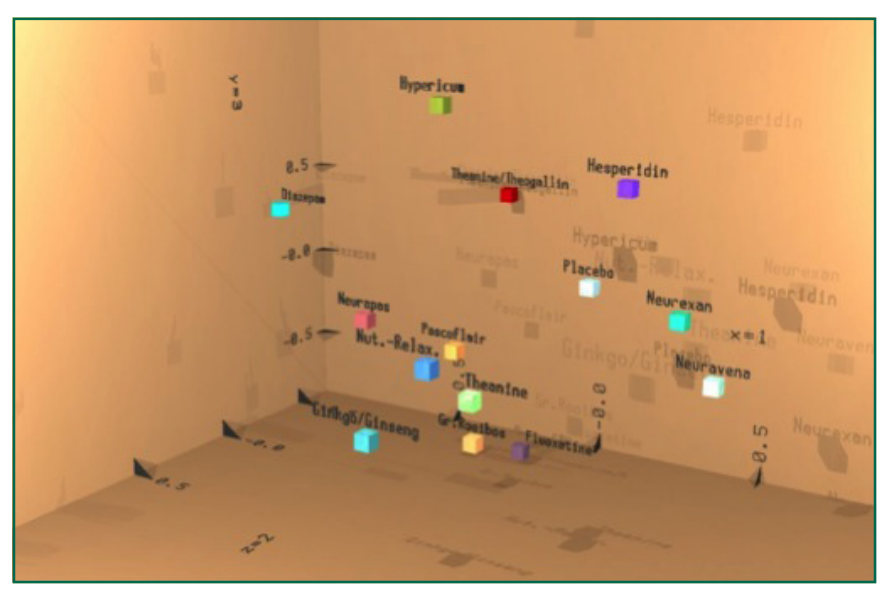

Abb. 3. Darstellung der unterschiedlichen Wirkung synthetischer wie auch pflanzlicher Präparate im Vergleich zu Placebo anhand einer Diskriminanzanalyse.

Die Erfassung der elektrischen Hirnaktivität in Form des Elektropharmakogramms stellt insgesamt eine probate Möglichkeit dar, um insbesondere die Wirkung von pflanzlichen Präparaten zu objektivieren. Ein Vergleich dieser Wirkungen kann besonders durch die Anwendung einer Diskriminanzanalyse erleichtert werden. Hierbei werden die Ergebnisse der ersten drei Funktionen in den Raum projiziert, die von weiteren drei Funktionen mithilfe einer additiven Farbmischung dargestellt werden (sog. RGB-mode). Ein repräsentatives Ergebnis wird in Abbildung 3 gezeigt:

Die Ableitung eines EEGs führt zum Einen zu einer Art von Bestandsaufnahme (Vergleich mit Hunderten von gesunden Gehirnen unserer Datenbank), zum Anderen zu einer rationalen Pharmakotherapie, insbesondere mit pflanzlichen Präparaten, wenn deren Wirkung im Rahmen eines Elektropharmakogramms vorher charakterisiert wurde.

\section{Weiterführende Literatur}

Dimpfel W: Enkephaloglyphen: Spektrale Signaturen der elektrischen Gehirntätigkeit als Spiegel der Psyche. Norderstedt, Books on Demand, 2011.

Dimpfel W, Koch K, Weiss G: Early effect of NEURAPAS ${ }^{\circledR}$ balance on current source density (CSD) of human EEG. BMC Psychiatry 2011;11:123.

Dimpfel W, Suter A: Sleep improving effects of a single dose administration of a valerian/hops fluid extract. A double blind, randomized, placebo-controlled sleepEEG study in a parallel design using the electrohypnogram. Eur J Med Res 2008; 13:200-204.

Schober F, Schellenberg R, Dimpfel W: Reflection of mental exercise in the dynamic quantitative topographical EEG. Neuropsychobiology 1995;31:98-112.

Todorova A, Hofmann H.C, Dimpfel W: A new frequency based automatic sleep analysis - description of the healthy sleep. Eur J Med Res 1997;2:85-197.

\section{Ginkgo und kognitive Störungen: Potenzial und Praxiserfahrung}

\section{Autor: Egemen Savaskan}

Die Komplexität der Pathologie der Demenz-Erkrankungen, die kognitive Beeinträchtigungen verursachen, macht die Entwicklung von Therapiestrategien zu einer Herausforderung. Obwohl die beiden herausragenden pathologischen Merkmale der Alzheimer-Demenz (AD), Amyloid-Peptid-Ablagerungen zu Plaques und Tau-Protein-assoziierte Zytoskelett-Veränderungen, im Fokus der Therapieforschung stehen, bieten andere pathologische Mechanismen, die zur Neurodegeneration beitragen, ebenfalls Ansätze für therapeutische Interventionen. Vor allem der oxidative Stress durch einen Überschuss der freien Radikale in den alternden Nervenzellen und Störungen in den Mitochondrien sind zusätzliche Pathologien, die Neurodegeneration fördern. Heute sind einige pflanzliche Substanzen bekannt, die eine starke antioxidative Wirkung vorweisen und in der Therapie der Demenz-Erkrankungen eine Rolle spielen können. Von diesen Substanzen ist vor allem der Ginkgo-biloba-Extrakt (EGb) sehr gut in präklinischen und klinischen Studien untersucht worden. Viele dieser Untersuchungen zeigen, dass EGb mitochondriale Atmung verbessert und oxidativen Stress reduziert [1]. Unter EGb sinken die freien Radikale im Gehirn und die neurotoxische Wirkung des Amyloid-Peptids wird reduziert.

Aufgrund dieser nachgewiesenen neuroprotektiven Eigenschaften wurde EGb in klinischen Studien in der Prävention und Therapie von AD untersucht. EGb ist bisher die einzige pflanzliche Substanz, die neben Antidementiva in die Therapieempfehlungen für Demenz-Erkrankungen aufgenommen wurde. In den Leitlinien der Weltföderation der Gesellschaften der Biologischen Psychiatrie ist EGb für die Therapie der AD und vaskulären Demenz empfohlen [2].

Der standardisierte EGb (EGb 761 $\left.{ }^{\circledR}\right)$ wurde in drei großen Studien für die Prävention der Demenz-Erkrankungen untersucht [3-5]. In der Placebo-kontrollierten Oregon-Studie, Dauer 3,5 Jahre, wurden 118 kognitiv gesunde Probanden über 85 Jahren mit $240 \mathrm{mg}$ EGb oder Placebo behandelt [3]. Personen, die eine hohe Adhärenz für Medikamenteneinnahme zeigten, wiesen einen geringeren kognitiven Abbau auf. In der zweiten Placebo-kontrollierten GEM-Studie wurden 3072 Probanden über 75 Jahren untersucht [4]. Sie waren entweder gesund oder zeigten beginnende kognitive Störungen. Die Dauer der Studie war 6,2 Jahre. In der Substanzgruppe trat deutlich weniger vaskuläre Demenz (VaD) auf. Gute Medikamenten-Adhärenz war auch in dieser Studie entscheidend. In der dritten Placebo-kontrollierten GuidAge-Studie wurden 2854 Probanden über 70 Jahren untersucht, die über Gedächtnisstörungen klagten [5]. Nach vierjähriger Behandlung mit EGb war die Inzidenz für AD halb so viel wie in der 
Placebo-Gruppe. Hier zeigte sich auch, dass kürzere Behandlungsdauer nicht den erwünschten Effekt aufweist.

Bei Personen zwischen 45 und 65 Jahren mit kognitiven Beschwerden verbesserten sich nach 12-wöchiger Behandlung mit EGb im Vergleich zu Placebo die Aufmerksamkeit und verzögerter Abruf im Wechsler-Memory-Test [6]. EGb $761^{\circledR}$ wurde auch in Placebo-kontrollierten Studien bei Patienten mit $\mathrm{AD}$ oder $\mathrm{VaD}$ untersucht [7-9]. In der GINDEM-Studie mit 214 AD- und 181 VaD-Patienten verbesserten sich die kognitiven Parameter, gemessen mit SKT, in der Behandlungsgruppe mit $240 \mathrm{mg}$ EGb im Vergleich zu Placebo nach 22 Wochen Therapie [7]. Ähnliche Resultate zeigte auch die GOTADAY-Studie bei 410 Patienten mit AD/VaD [8]. In der GOT-IT-Studie bei 402 Patienten mit AD/VaD wies man nach 24-wöchiger Behandlung mit EGb Verbesserungen im SKT, Aktivitäten des täglichen Lebens und verbaler Flüssigkeit nach [9]. Ein wichtiger Befund all dieser drei Studien war eine zusätzliche positive Wirkung von EGb auf die neuropsychiatrischen Begleitsymptome der Demenz. Diese Wirkung wurde mit NPI (Neuropsychiatric Inventory) gemessen. Die Auswertung der NPI-Resultate zeigte, dass vor allem die Symptome Apathie/Indifferenz, Schlaf-WachRhythmus-Störungen, Irritabilität, Depression und motorische Unruhe von der Behandlung mit EGb profitierten [10]. Ein Nebenbefund der Untersuchung war, dass es bei den Betreuern der Patienten zu einer Abnahme des Stresses parallel zur Verbesserung der neuropsychiatrischen Begleitsymptome und der Aktivitäten des täglichen Lebens kam. Dieser Befund wurde in allen Studien mit AD und VaD bestätigt.

In all den oben erwähnten Studien war die Verträglichkeit von EGb gut, oft vergleichbar mit der Placebo-Gruppe. Selten traten Übelkeit, Kopfschmerzen, Schlaflosigkeit, Unruhe, Konfusion oder Hautreaktionen auf. Die Studienabbruchrate aufgrund von Nebenwirkungen war deutlich geringer als in Studien mit anderen Antidementiva [11]. Insgesamt kann man zusammenfassend sagen, dass EGb bei kognitiven und neuropsychiatrischen Symptomen der Demenz wirksam ist. Eine hohe Therapieadhärenz und lange Therapiedauer sind allerdings für die Wirksamkeit Voraussetzungen.

\section{Literatur}

1 Eckert A: Mitochondrial effects of Ginkgo biloba extract. Int Psychogeriatr 2012; 24(suppl 1):18-20.

2 World Federation of Societies of Biological Psychiatry (WFSBP): Guidelines for the biological treatment of Alzheimer's disease and other dementias. World J Biol Psychiatry 2011;12:2-32.

3 Dodge HH, Zitzelberger T, Oken BS, et al: A randomized placebo-controlled trial of Ginkgo biloba for the prevention of cognitive decline. Neurology 2008;70: 1809-1817.

4 DeKosky ST, Williamson JD, Fitzpatrick AL, et al: Ginkgo biloba for prevention of dementia. A randomized controlled trial. JAMA 2008;300:2253-2262.

5 Vellas B, Coley N, Ousset P-J, et al: Long-term use of standardised ginkgo biloba extract for the prevention of Alzheimer's disease (GuideAge): a randomized placebo-controlled trial. Lancet Neurol 2012;11:851-859.

6 Grass-Kapanke B, Busmane A, Lasmanis A, et al: Effects of Ginkgo biloba extract EGb $761^{\circledR}$ in very mild cognitive impairment (vMCI). Neurosci Med 2011;2:48-56.
7 Napryeyenko O, Sonnik G, Tartakovsky I: Efficacy and tolerability of Ginkgo biloba extract EGb $761^{\circledR}$ by type of dementia: analyses of a randomised controlled trial. J Neurol Sci 2009;283:224-229.

8 Ihl R, Bachinskaya N, Korczyn AD, et al: Efficacy and safety of a once-daily formulation of Ginkgo biloba extract EGb 761 in dementia with neuropsychiatric features: a randomized controlled trial. Int J Geriatr Psychiatr 2011;26:1186-1194.

9 Herrschaft H, Nacu A, Likhachev S, et al: Ginkgo biloba extract EGb $761^{\circledR}$ in dementia with neuropsychiatric features: a randomised, placebo-controlled trial to confirm the efficacy and safety of a daily dose of $240 \mathrm{mg}$. J Psychiatr Res 2012;46:716-723.

10 Bachinskaya N, Hoerr R, Ihl R: Alleviating neuropsychiatric symptoms in dementia: the effects of Ginkgo biloba extract EGb $761^{\circledR}$. Findings from a randomized controlled trial. Neuropsychiatr Dis Treat 2011;7:209-215.

11 Kasper S, Schubert H: Ginkgo-Spezialextrakt EGb $761^{\circledR}$ in der Behandlung der Demenz: Evidenz für Wirksamkeit und Verträglichkeit. Fortschritte der Neurologie. Psychiatrie 2009;77:494-506.

\section{Heilkräuter in der Gartentherapie}

\section{Autor: Thomas Pfister}

Gartentherapie ist im deutschsprachigen Raum noch wenig bekannt, obwohl sie bereits in den 1950er-Jahren des letzten Jahrhunderts in den USA entwickelt worden ist. Verschiedene Forschungsergebnisse bestätigen die positiven Wirkungen der Gartentherapie auf Körper und Psyche. Der Einsatz von Heilkräutern in der Gartentherapie ist besonders erfolgversprechend, weil neben den allgemeinen Wirkungen der Gartentherapie die positiven Effekte der Heilkräuter einen zusätzlichen Benefit bringen.

\section{Heilende Gärten}

Die heilende Wirkung von Gärten wurde bereits vor langer Zeit entdeckt. So sollen ägyptische Ärztinnen und Ärzte ihren Patientinnen und Patienten Gartenspaziergänge empfohlen haben, um die Genesung zu fördern. Moderne wissenschaftliche Studien zeigen, dass nur schon ein Blick ins Grüne bei Patientinnen und Patienten schmerzlindernd wirken kann. Die heilende Wirkung von Gartenarbeit entdeckte man in der Psychiatrie bereits im 18. Jahrhundert. Die Gartentherapie («horticultural therapy») wurde in den 1950er-Jahren von US-amerikanischen Fachleuten bei der Rehabilitation von Soldaten aus dem Korea- und Vietnamkrieg entwickelt. Sowohl körperliche Verletzungen als auch psychische Traumata können mit Aktivitäten im Garten besser und schneller geheilt werden.

\section{Definition von Gartentherapie}

«Gartentherapie ist ein von einer ausgebildeten Gartentherapeutin oder einem Gartentherapeuten gesteuerter Prozess, in welchem Pflanzen und Gartenaktivitäten gezielt dazu verwendet werden, das soziale, psychische und körperliche Wohlbefinden von Menschen zu stärken» [1]. Pflanzen und andere 
Naturaktivitäten dienen als Mittel, um bei Patientinnen und Patienten mit unterschiedlichen Diagnosen therapeutische Ziele zu erreichen.

Man kann zwischen drei Ansätzen von Gartentherapie unterscheiden:

Zum Ersten wird sie in der Arbeitsagogik eingesetzt und bezweckt die Wiedereingliederung einer Person in den Arbeitsprozess. So können gärtnerische Tätigkeiten im Rahmen einer psychiatrischen Behandlung zur Belastungserprobung eingesetzt werden.

Zum Zweiten wird Gartentherapie therapeutisch angewendet, um eine Patientin oder einen Patienten mit einer klaren Diagnose bei der Heilung zu unterstützen. Ein Beispiel dafür sind gartentherapeutische Programme bei der Rehabilitation von hirnverletzten Personen, die deren Feinmotorik fördern.

Zum Dritten wird Gartentherapie zur Förderung der Lebensqualität und der Gesundheit eingesetzt. Menschen in einer Institution bekommen so die Möglichkeit, im Garten aktiv zu werden.

Gartentherapie kann in vielen Bereichen eingesetzt werden, so zum Beispiel in Alters- und Pflegezentren, Behinderteninstitutionen, psychiatrischen Einrichtungen, Sonderschulen, Krankenhäusern oder Rehabilitationskliniken.

\section{Wirkungen der Gartentherapie}

Seit den siebziger Jahren des letzten Jahrhunderts gibt es viele Forschungsergebnisse, welche die positiven Wirkungen von Gartentherapie nachweisen. So wurde bei Personen, die in Gartentherapieprogrammen mitmachten, eine erhöhte Schmerztoleranz sowie eine verminderte subjektiv empfundene Schmerzwahrnehmung festgestellt. Andere Studien stellten eine Reduktion von Stress fest und verminderte Gefühle von Ärger und Aggressionen. Forschungsergebnisse belegen, dass durch Gartentherapie neben einer Verbesserung des allgemeinen Gemütszustandes auch ein erhöhter Selbstwert sowie eine Steigerung von positiven Emotionen erzielt werden können. Im Bereich Herz-Kreislauf sind positive Effekte wie z.B. eine erniedrigte Herzfrequenz oder ein vermindertes Risiko für koronare Herzkrankheiten zu beobachten. Schließlich können mit gartentherapeutischen Interventionen die Konzentrati- onsfähigkeit erhöht und Schlaftiefe und -qualität verbessert werden.

\section{Therapiegärten}

Für die Gartentherapie werden spezielle Gärten angelegt, die je nach Klientel unterschiedlich gestaltet sind. Zentral ist eine möglichst vielseitige Verwendung. Der Garten dient als eine Art «Werkzeugkasten», der von der Gartentherapeutin bzw. vom Gartentherapeuten gezielt eingesetzt wird. Im Zentrum stehen die verschiedenen Pflanzen, die möglichst alle Sinne auf unterschiedliche Weise anregen sollen. In vielen Therapiegärten stehen Hochbeete, um den Benutzerinnen und Benutzern die Gartenarbeit zu erleichtern. Obstbäume, Beerensträucher und Gemüsebeete werden für die Erzeugung von Nahrungsmitteln benutzt, wobei diese heute weniger Bedeutung haben als früher.

\section{Gartentherapie mit Heilkräutern}

Für die Gartentherapie können mit Ausnahme von Giftpflanzen fast alle Pflanzen verwendet werden. Bei älteren Personen sind zum Beispiel Gemüse und Früchte sehr beliebt, da sie diese früher oft selber im Garten angebaut haben. Oft werden auch Schnittblumen kultiviert, die dann in den Institutionen als schöne Dekoration verwendet werden.

Besonders interessant und in der Gartentherapie vielseitig nutzbar sind Heil- und Küchenkräuter. Obwohl die alten Kräuterbücher von Künzle, Madaus und Vogel kaum mehr in der Hausbibliothek zu finden sind, werden viele Kräuter noch regelmäßig in der Küche verwendet, sei es als Gewürzkraut wie z.B. Petersilie, Liebstöckel oder Oregano oder als Nahrungsmittel wie z.B. Zwiebel, Erdbeere oder Brombeere.

Viele Heilkräuter regen mit ihren Inhaltsstoffen unsere Sinne an. Gerade Pflanzen mit ätherischen Ölen wie zum Beispiel Lavendel, Zitronenmelisse, Minze, Kamille, Thymian bescheren intensive Dufterlebnisse. Jede Pflanze hat ihren spezifischen Charakter und löst je nach Person bestimmte Gefühle aus. Dies wird auch im Rahmen der Aromatherapie benutzt.
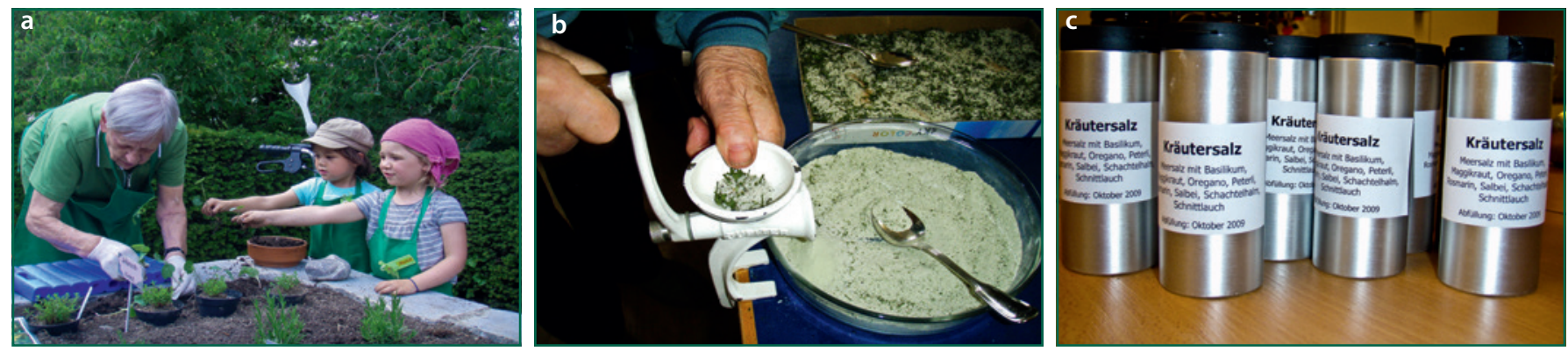

Abb. 1. a Jung und Alt beim gemeinsamen Gärtnern. b, c Kräutersalzherstellung. Fotos: Thomas Pfister. 
Die Kräuter im Garten haben den Vorteil, dass sie sich leicht anbauen und vielseitig verwenden lassen. Besonders beliebt in der Gartentherapie ist die Herstellung von Kräutersalz. Gewürzpflanzen wie z.B. Majoran, Oregano oder Schnittlauch werden getrocknet und dann zusammen mit Meersalz gemahlen in Salzstreuer eingefüllt (Abb. 1).

Andere Heilkräuter bzw. deren Früchte lassen sich zu Konfitüren oder Chutneys verarbeiten. Besonders beliebt in Gartengruppen ist die Herstellung von Sirup, zum Beispiel aus Holunderblüten, Melissen- oder Pfefferminzblättern.

Erste Alterseinrichtungen setzen die im eigenen Garten angepflanzten Heilkräuter in der Phytotherapie ein. Im Rahmen der Pflege werden die Teemischungen bei allerlei Beschwerden wie Verdauungsproblemen oder Atemwegserkrankungen erfolgreich eingesetzt [2].

\section{Fazit}

Mit Heilkräutern in der Gartentherapie erzeugt man eine doppelte Wirkung: Zum einen trägt die Gartenarbeit dazu bei, die eigene Gesundheit zu erhalten und zu fördern; zum anderen kann man die selber erzeugten Heilkräuter genießen oder als Hausmittel bei alltäglichen Beschwerden einsetzen.

\section{Literatur}

1 Niepel A, Pfister T: Praxisbuch Gartentherapie. Idstein, Schulz-Kirchner, 2010.

2 Pfister, T Saller R, et al: Heilkräuter im Garten - pflanzen, ernten, anwenden. Bern, Haupt, 2014

\section{Das Potential der Phytotherapie bei Erkrankungen der Prostata mit Schwerpunkt Prostatakarzinom}

\section{Autor: Matthias Rostock}

Erkrankungen der Prostata spielen bei Männern in der westlichen Welt mit steigendem Alter eine zunehmend dominierende Rolle. So ist die benigne Prostatahyperplasie (BPH) der häufigste gutartige Tumor des Mannes und betrifft bis zu $40 \%$ in der Altersgruppe ab dem 50. Lebensjahr.

\section{Benigne Prostatahyperplasie}

Die Vergrößerung der Prostata für sich allein ist noch keine Therapieindikation, wenn keine hiermit im Zusammenhang stehenden Beschwerden beobachtet werden. Wir sprechen heute von dem Begriff Benignes Prostatasyndrom, wenn bei einer gutartigen Prostatavergrößerung Beschwerden des unteren Harntrakts bestehen wie ein abgeschwächter Harnstrahl, eine verzögerte Harnentleerung, ein Nachträufeln sowie ein Restharngefühl. Zusätzlich leiden die Patienten häufig unter Pollakisurie, Nykturie, Algurie und wiederholtem imperativem Harndrang, bei zunehmender Symptomatik mit Urge-Inkontinenz. Im Rahmen der Erstdiagnose sollte neben Anamnese, objektivierbarer Einschätzung der Beschwerdeintensität (z.B. mit dem internationalen Prostata-Symptomen-Score; IPSS), körperlicher Untersuchung, Urinstatus, differentialdiagnostischer Bestimmung des PSA-Wertes auch an die sonographische Untersuchung von Prostata und Harnblase und möglichst auch eine Uroflowmetrie gedacht werden. Vor einer differentialtherapeutischen Auseinandersetzung mit den medikamentösen Therapieoptionen sollte zunächst ausgeschlossen werden, ob bei nachgewiesener Obstruktion durch eine benigne Prostatahyperplasie eine absolute Indikation für ein operatives Vorgehen vorliegt (wiederholtes Vorliegen eines akuten Harnverhalts, wiederholtes Auftreten einer Makrohämaturie, das Vorliegen von Harnblasensteinen, chronisch rezidivierende Harnwegsinfektionen, Stauungszeichen für die oberen Harnwege sowie eine eingeschränkte Nierenfunktion).

Zur medikamentösen Therapie des benignen Prostatasyndroms findet man in den Leitlinien zu den Alpha-1-Rezeptorantagonisten, 5-alpha-Reduktase-Hemmern und Muskarinrezeptorantagonisten sowie ihren Kombinationen dezidierte Angaben für ihren differentialtherapeutischen Einsatz. Die Phytotherapeutika werden erwähnt, aber in ihrer therapeutischen Wirksamkeit als im Großen und Ganzen aufgrund der in den bisher durchgeführten Therapiestudien gewonnenen Erkenntnisse als nicht gut einschätzbar gewertet. Auf Patientenseite ist der Bedarf nach einer phytotherapeutischen Behandlung ihrer Beschwerden aber nach wie vor ungebrochen mit einem geschätzten Marktanteil von 20-40\%, ohne dass bei dieser Berechnung die sog. OTC-Präparate berücksichtigt worden wären [1]. Nicht zuletzt gründet sich dies auf eine häufig deutlich schlechtere Verträglichkeit der synthetischen Präparate mit Abgeschlagenheit, Schwindel, Kopfschmerzen, Diarrhö und Blutdruckabfall (Alpha-1-Rezeptorantagonisten), Libidoverlust, erektiler Dysfunktion und Schwellung der Brustdrüse (5-alpha-Reduktase-Hemmer) sowie Verwirrtheit, Schlaf- und Gedächtnisstörungen und erhöhtem Risiko für einen akuten Harnverhalt (Muskarinrezeptorantagonisten).

Anwendung finden in der Phytotherapie vor allem Extrakte aus Sägepalmenfrüchten (Serenoa repens oder Sabal serrulata), Brennnesselwurzeln (Urtica dioica), der Rinde des afrikanischen Pflaumenbaumes (Pygeum africanum oder Prunus africana), Samen des Kürbisses (Cucurbita pepo) sowie Roggenpollen (Secale cereale) und Phytosterolextrakte in Form von Mono- oder Kombinationspräparaten. Für die genannten Extrakte liegen positive Ergebnisse auf die subjektive Beschwerdesymptomatik im Zusammenhang mit einer benignen Prostatahyperplasie aus einer Vielzahl von kontrollierten Therapiestudien vor, die in einer Reihe systematischer Reviews zusammengefasst worden sind. Allerdings sind in den letzten Jahren die Anforderungen an die Forschungsmethodik vonseiten der 
internationalen WHO-BPH-Konsensuskonferenzen erheblich gestiegen u.a. bezüglich einer Studiendauer von mindestens 12 Monaten. Aktuelle Studienergebnisse konnten vor diesem Hintergrund z.T. die Resultate früherer Untersuchungen nicht bestätigen.

\section{Prostatitis und chronisches Beckenschmerz-Syndrom}

Die akute bakterielle Prostatitis, die mit Fieber, schwerem Krankheitsgefühl und häufig starken lokalen Beschwerden auftritt, ist eine Indikation für die sofortige und hochdosierte gezielte antibiotische Therapie. Auch die chronische bakterielle Prostatitis sollte gezielt, z.B. nach Ejakulat-Kultur, antibiotisch behandelt werden. Die Abgrenzung Letzterer von einer chronischen abakteriellen Prostatatitis (Kongestationsprostatitis) oder einem chronischen nicht entzündlichen Schmerzsyndrom des Beckens (Chronic Pelvic Pain Syndrome, CPPS), ist von wesentlicher Bedeutung für das therapeutische Vorgehen. Während bei der bakteriellen Infektion selbstverständlich die antibiotische Behandlung im Vordergrund steht, handelt es sich bei Letzterem um ein komplexes psychosomatisches Krankheitsbild. Die Symptomatik reicht von einer unspezifischen Schmerzsymptomatik im Dammbereich, z.T. mit Ausstrahlung in die Hoden, z.T. mit Spannungsgefühl im Kreuzbeinbereich. Dabei bestehen Beschwerden des unteren Harntrakts in Form von vermehrtem Harndrang, erschwertem und schmerzhaftem Wasserlassen mit Nachträufeln und Restharngefühl. Häufig leiden die Patienten zusätzlich unter Libidoverlust und erektiler Dysfunktion sowie Schmerzem beim Geschlechtsverkehr. Diagnostisch müssen organische Ursachen für die Beschwerden ausgeschlossen werden. Im Vordergrund der Behandlung stehen Psychotherapie und Entspannungsübungen, lokale Wärme, aber auch ordnungstherapeutische Aspekte u.a. hinsichtlich eines förderlichen Ernährungs- und Bewegungskonzeptes. In der phytotherapeutischen Behandlung liegen erste Studienergebnisse mit Roggenpollenextrakt [2] sowie auch mit einem Quercetin-haltigen Präparat [3] vor. Aber auch eine Behandlung mit psychotrop wirksamen Pflanzenextrakten, z.B. mit einer Kombination aus Pestwurzwurzel-, Baldrianwurzel-, Passionsblumenkraut- und Melissenblätterextrakt, könnte sich bei diesem somatoformen Beschwerdekomplex als hilfreich erweisen [4]. Andere komplementärmedizinische Ansätze wie Akupunktur, Triggerpunktmassage oder auch komplexe Interventionen im Rahmen der Mind-Body-Medizin könnten in der Behandlung hilfreich kombiniert werden [5].

\section{Prostatakarzinom}

Das Prostatkarzinom ist der häufigste maligne Tumor des Mannes in der westlichen Welt. Die Behandlung erfolgt in den meisten Fällen potentiell kurativ mittels radikaler Prostatektomie oder kurativ intendierter Strahlentherapie. Bei lokal fortgeschrittenem oder metastasiertem Befund besteht die Indikation zur Hormontherapie und im Verlauf bei hormonrefraktärem Zustand zu einer chemotherapeutischen Behandlung mit palliativer Zielrichtung. In verschiedenen Phasen der Erkrankung kann darüber hinaus eine phytotherapeutische Behandlung als begleitende oder auch als alleinige Massnahme hilfreich für den Patienten sein:

Hochgradige intraepitheliale Prostata-Neoplasie (HGPIN)

Eine hochgradige intraepitheliale Prostata-Neoplasie wird nicht selten diagnostiziert, wenn bei Patienten wegen eines erhöhten PSA-Wertes Prostata-Biopsien durchgeführt werden, aber noch kein invasives Prostatakarzinom vorliegt. Patienten mit diesem Befund haben ein deutlich erhöhtes Risiko für das Auftreten eines invasiven Prostatakarzinoms - es gibt aber bis heute keine etablierte Strategie zur Prävention. In dieser Situation wurde in einer italienischen prospektiv randomisierten Doppelblindstudie mit 60 Patienten der Therapieeffekt eines Grüntee-Eextraktes $(3 \times$ tgl. $1 \mathrm{Kps}$. mit $200 \mathrm{mg}$ Grüntee-Extrakt) gegenüber Placebo untersucht. Während einer Therapiephase von 12 Monaten wurde in der Placebo-behandelten Kontrollgruppe bei insgesamt neun von 30 Patienten ein invasives Prostatakarzinom mittels Kontrollbiopsie diagnostiziert, während dies in der Grüntee-Extrakt-Gruppe nur bei einem von 30 Patienten der Fall war [6]. Der statistisch signifikante Unterschied dieser Studie wurde zwei Jahre später in einer Follow-up-Untersuchung bestätigt. Derzeit wird eine größere nachfolgende Phase-III-Studie durchgeführt, deren Ergebnisse abgewartet werden müssen.

\section{PSA-Rezidiv nach kurativ intendierter Primärtherapie}

Wenn es nach erfolgter Primärbehandlung des invasiven Prostatakarzinoms z.B. mittels Operation im weiteren Verlauf $\mathrm{zu}$ einem erneuten Ansteigen des PSA-Wertes kommt, geht man von dem Vorliegen eines Rezidivs aus. In einer ersten unkontrollierten Phase-II-Studie, die in Kalifornien durchgeführt worden war, hatten 46 Männer mit einem PSA-Rezidiv (nach mittels OP oder Strahlentherapie erfolgter Primärbehandlung) ihres Prostatakarzinoms $240 \mathrm{ml}$ Granatapfelsaft mit einem Gehalt an $570 \mathrm{mg}$ Polyphenolen täglich eingenommen. Hierunter kam es zu einer signifikanten Verlängerung der PSA-Verdopplungszeit von 15 auf 54 Monate [7]. In einer nachfolgenden randomisierten Studie mit $\mathrm{n}=104$ Patienten in vergleichbarer Situation wurde jeweils entweder $1 \mathrm{~g}$ oder $3 \mathrm{~g}$ Granatapfel-Extrakt (entsprechend einem Polyphenolgehalt von 1000 mg bzw. 3000 mg) verabreicht. In beiden Gruppen stieg unter der Medikation die PSA-Verdopplungszeit ebenfalls signifikant im Vergleich zum Baseline-Wert an, der Dosis-Unterschied hatte allerdings keine Auswirkung auf den Therapieeffekt [8]. Dies sind zunächst durchaus vielversprechende Ergebnisse. Es müssen aber weitere kontrollierte Studien folgen, um den klinischen Stellenwert von Granatapfel-Extrakt in dieser Situation eindeutiger bewerten zu können. Entsprechende Projekte, auch mit Kombinationen 
pflanzlicher Extrakte laufen derzeit in verschiedenen Ländern. Als wichtige Limitierung für den Einsatz komplementärer Therapiemaßnahmen bei Slow-rising-PSA ist in jedem Falle $\mathrm{zu}$ bedenken, dass in dieser Situation eine strahlentherapeutische Behandlung sekundär kurativ wirksam sein kann, was bedeutet, dass zumindest ein Teil der Patienten hierdurch langfristig tumorfrei bleiben wird. Wenn mit der Strahlentherapie zu lange zugewartet wird, sinkt aber die Ansprechrate, so dass in der aktuellen S3-Leitlinie zum Prostatakarzinom empfohlen wird, in dieser Situation den PSA-Wert nicht über $0,5 \mathrm{ng} / \mathrm{ml}$ ansteigen zu lassen.

Eine weitere Placebo-kontrollierte Studie aus England suchte in einer ähnlichen Situation bei $n=203$ Patienten mit PSA-Rezidiv nach abgeschlossener Primärbehandlung nach einem Therapieeffekt durch ein Kombinationspräparat mit Granatapfel-, Grüntee-, Brokkoli- und Curcuma-Extrakt. Auch hier kam es gegenüber der Placebo-Gruppe zu einer Verzögerung des PSA-Anstiegs bzw. Rückgang des PSA-Wertes [9]. Möglicherweise ist eine Kombinationsbehandlung mit phytotherapeutischen Extrakten, die teils unterschiedliche, teils überschneidende Zielstrukturen haben, noch effektiver als eine Monotherapie mit Einzelextrakten [10]. Diese ersten wissenschaftlichen klinischen Daten lassen sich mit Vorsicht durchaus auch auf die häufige Situation der «Active Surveillance» und des «Watchful waitings» beziehen, entsprechende Therapiestudien befinden sich in Durchführung.

\section{Lokal fortgeschrittenes und/oder metastasiertes \\ Prostatakarzinom}

Bei fortgeschrittener Tumorerkrankung kommt es im weiteren Krankheitsverlauf bei dem einen Patienten sehr früh, bei dem anderen Patienten nach langem Intervall zu einer zunehmenden Resistenz gegen die anfangs sehr wirkungsvolle hormontherapeutische Behandlung seines Prostatakarzinoms. Auch in dieser Situation kann der Einsatz von Phytotherapeutika hilfreich sein. In den vergangenen Jahren wurden häufiger phytotherapeutische Kombinationspräparate über das Internet vertrieben, unter deren Einsatz beeindruckende PSA-Rückgänge und teilweise auch deutliche Beschwerdebesserungen berichtet wurden. Leider wurden wiederholt nicht deklarierte Beimengungen synthetischer Wirkstoffe wie Diethylstilbestrol oder Diclofenac in diesen Präparaten gefunden; entsprechend ist die Verunsicherung bei Patienten und Verordnern gross. In der Schweiz wurde vor diesem Hintergrund im Kanton Schwyz eine Magistralrezeptur entwickelt, die eine derartige Wirkstoffkombination zumindest offiziell deklariert und damit auf einen legalen Boden stellt [11] - dies kann dann allerdings nicht mehr originär als Phytotherapie bezeichnet werden.

Die Mistel (Viscum album) ist die im deutschen Sprachraum am häufigsten von Patienten mit einer Tumorerkrankung eingesetzte Heilpflanze, insbesondere in fortgeschrittener Therapiesituation. Mistelextrakt wird zwei- bis dreimal wöchentlich s.c. injiziert, i.d.R. ergänzend zur konventionellen Behandlung.
Beim Prostatakarzinom liegen derzeit keine Ergebnisse aus vergleichenden Therapiestudien zur Misteltherapie vor, die für sich allein eine Empfehlung wissenschaftlich solide begründen würden. Die Hauptindikation der Mistel in der komplementären Krebstherapie ist jedoch die Begleitbehandlung zur konventionellen Therapie mit dem Ziel, hierdurch eine Verbesserung ihrer Verträglichkeit, eine Minderung ihrer Nebenwirkungen und eine Optimierung der Lebensqualität der Patienten erreichen zu können. Einem Review der Cochrane Collaboration zur Misteltherapie zufolge konnte dies in der Mehrzahl der hierzu durchgeführten Therapiestudien zumindest in Teilbereichen erzielt werden. Es wird aber auf methodische Schwächen in einem größeren Anteil der Studien hingewiesen [12]. Die meisten Daten sind außerdem im Zusammenhang mit nichturologischen Tumorerkrankungen erhoben worden. Diese lassen sich jedoch bei vorsichtiger Handhabung zumindest teilweise u.U. auch auf die Situation von Patienten mit einem Prostatakarzinom übertragen. Trotz anhaltender kontroverser Diskussionen in der Onkologie zählt Mistelextrakt im deutschsprachigen Raum zu den pflanzlichen Arzneimitteln in der Komplementärmedizin, deren Kosten von den Krankenversicherungen übernommen werden, in Deutschland bezieht sich dies zumindest auf die palliative Therapiesituation.

\section{Literatur}

1 Wehrberger C, Dreikorn K, Schmitz-Dräger BJ, Oelke M, Madersbacher S: Phytotherapie bei benignem Prostatasyndrom und Prostatakarzinom. Urologe 2012;51:1674-1682.

2 Wagenlehner FME, Schneider H, Ludwig M, Schnitker J, Brähler E, Weidner W: A pollen extract (Cernilton) in patients with inflammatory chronic prostatitis-chronic pelvic pain syndrome: a multicentre randomised, prospective, double-blind, placebo-controlled phase 3 study. Eur Urol 2009;56:544-551.

3 Shoskes DA, Zeitlin SI, Shahed A, Rajfer J: Quercetin in men with category III chronic prostatitis: a preliminary prospective, double-blind, placebo-controlled trial. Urology 1999;54:960-963.

4 Melzer J, Schrader E, Brattström A, Schellenberg R, Saller R: Fixed herbal drug combination with and without butterbur (Ze 185) for the treatment of patients with somatoform disorders: randomized, placebo-controlled pharmacoclinical trial. Phytother Res 2009;23:1303-1308.

5 Herati S, Moldwin RM:Alternative therapies in the management of chronic prostatitis/chronic pelvic pain syndrome. World J Urol 2013;31:761-766.

6 Bettuzzi S, Brausi M, Rizzi F, Castagnetti G, Peracchia G, Corti A: Chemoprevention of human prostate cancer by oral administration of green tea catechins in volunteers with high-grade prostate intraepithelial neoplasia: a preliminary report from a one-year proof-of-principle study. Cancer Res 2006;66:1234-1240.

7 Pantuck AJ, Leppert JT, Zomorodian N, Aronson W, Hong J, Barnard RJ, Seeram N, Liker H, Wang H, Elashoff R, Heber D, Aviram M, Ignarro L, Belldegrun A: Phase II study of pomegranate juice for men with rising prostate-specific antigen following surgery or radiation for prostate cancer. Clin Cancer Res 2006;12:4018 4026.

8 Paller CJ, Wozniak PJ, Gillespie BK, Sieber PR, Greengold RH, Stockton BR, Hertzman BL, Efros MD, Reper RP, Liker HR, Carducci MA: A randomized phase II study of pomegranate extract for men with rising PSA following initial therapy for localized prostate cancer. Prostate Cancer Prostatic Dis 2013;16:5055.

9 Thomas R, Williams M, Sharma H, Chaudry A, Bellamy P: A double-blind, placebo-controlled randomized trial evaluating the effect of a polyphenol-rich whole food supplement on PSA progression in men with prostate cancer - the UK NCRN Pomi-T study. Prostate Cancer Prostatic Dis 2014;DOI:10.1038/pcan.2014.6.

10 Saller R, Rostock M: Multimorbidität und Multi-Target-Therapie in der Phytotherapie. Praxis (Bern 1994);101:1637-1642. 
11 Schuermann M: Östrogenbasierte Phytotherapie beim Prostatakarzinom Schweiz Z Ganzheitsmed 2011;23:267-271.

12 Horneber M, Bueschel G, Huber R, Linde K, Rostock M: Mistletoe therapy in oncology. Cochrane Database Syst Rev 2008;CD003297.

\section{Komplementärmedizin bei Brustkrebs - Evaluation des Pilotprojekts in Meran}

\section{Autoren: Claudia M. Witt, Christian Thuile}

Die Evidenz zur integrativen Onkologie beschränkte sich zumeist auf die Bewertung von Einzelmaßnahmen in definierten Settings. Die Routineversorgung findet aber in einem komplexen Kontext mit sehr individuellen Patienten statt. Entsprechend haben Ärzte und Patienten ebenso wie Kostenund Entscheidungsträger häufig erhebliche Mühe, aus der Evidenzlage Schlussfolgerungen für einen konkreten Einzelfall zu ziehen [1]. Entscheidungsträger benötigen demzufolge Studien, die Therapiealternativen direkt vergleichen, patientenrelevante Ergebnisparameter verwenden und unter meist weniger standardisierten Alltagsbedingungen ablaufen [2]. Für diese Art von Forschung hat sich in den letzten Jahren im angelsächsischen Raum eine Bezeichnung etabliert: Comparative Effectiveness Research (CER). Das amerikanische Institute of Medicine hat CER wie folgt definiert: «CER ist die Erzeugung und Synthese von Evidenz, welche Nutzen und Risiko alternativer Methoden zur Prävention, Diagnostik, Behandlung oder Steuerung einer Erkrankung oder zur Verbesserung der Gesundheitsversorgung vergleicht. Das Ziel von CER ist es, Verbraucher, Ärzte, Versicherer und politische Entscheidungsträger darin zu unterstützen, informierte Entscheidungen zu treffen, die die Gesundheitsversorgung auf beiden Ebenen verbessern, der individuellen und der Bevölkerungsebene» [3].

Dieser Anspruch wurde im Rahmen der Evaluierung des Pilotprojekts zur Komplementärmedizin (Beschluss der Landesregierung Nr. 37/2009) am Krankenhaus Meran umgesetzt. Primäres Ziel war die Evaluation der vergleichenden Effektivität einer zusätzlichen komplementärmedizinischen Behandlung versus alleiniger konventioneller Behandlung bei Patientinnen mit Mammakarzinom unter Bedingungen der Routineversorgung. Die Studienergebnisse sollten die politische Entscheidung bezüglich der Fortsetzung des Projektes am Krankenhaus Meran informieren.

Erste Ergebnisse wurden auf der Tagung der International Society for Complementary Medicine Research (ISCMR) 2013 in London präsentiert (s. Box 1).

Im Rahmen der Studie wurde eine komplexe und individualisierte komplementärmedizinische Therapie angewendet, die unter der Verantwortung des Leiters des Pilotprojektes (Christan Thuile) entwickelt und angewendet wurde. Neben anderen Verfahren spielte auch die Phytotherapie eine rele-

\section{Box 1:}

Background: Because patients with cancer often seek complementary medicine, the Department of Health in South Tyrol (Italy) established a service for complementary medicine at the Hospital of Meran. The aim of this study was to evaluate the effectiveness of an additional, individualized, multi-component complementary medicine treatment offered at the Hospital Meran on health-related quality of life compared to usual care only in patients with breast cancer.

Methods: A randomized pragmatic trial with two parallel arms was performed. Women with confirmed diagnoses of breast cancer treated in one of three breast cancer centers (Bozen, Brixen, Meran) were randomized (stratified by the usual care treatment during the study) to receive individualized complementary medicine (CM group) or to have no additional complementary medicine (usual care group). Both groups were allowed to use conventional usual care treatments. Primary endpoint was the quality of life FACT-B score at 6 months. For statistical analysis we used analysis of covariance (with factors treatment and stratum, and baseline FACT-B score) and imputed missing FACT-B scores at 6 months with regression based multiple imputation.

Results: A total of 275 patients were randomized between April 2011 and March 2012 to the CM group $(n=136,56.3 \pm 10.9$ years of age) or the usual care group $(n=139,56.0 \pm 11.0)$. After 6 months adjusted means for health-related quality of life were higher in the CM group (FACT-B score 107.94, 95\% Cl 104.04$111.83)$ compared to the usual care group (102.13, 98.47105.79) with an adjusted FACT-B score difference between groups of $5.80(2.72-8.89, \mathrm{p}=0.0002)$.

Conclusion: An additional individualized and complex complementary medicine intervention was more effective in improving quality of life than no additional complementary medicine intervention in breast cancer patients. Further studies evaluating specific effects of treatment components should follow.

Trial Registration: ClinicalTrials.gov registration number: NCT01458457

Witt C, Außerer O, Baier S, Heidegger H, Mayr O, Mitterer M, Roll S, Spizzo G, Scherer A, Thuile C, Wieser A, Schützler L: Effectiveness of an additional individualized multi-component complementary medicine treatment on health-related quality of life in breast cancer patients: A pragmatic randomized trial. Forsch Komplementmed 2013;20(suppl 1):45.

vante Rolle und soll im Folgenden detaillierter dargestellt werden. Patientinnen erhielten im Rahmen dieser Studie die Phytotherapie symptombezogen als Begleittherapie angeboten, mit dem Ziel Nebenwirkungen der konventionellen Therapien zu minimieren. Dabei wurde darauf geachtet, Wechselwirkungen möglichst zu vermeiden.

In Box 2 sind die im Rahmen der Studie häufigen Indikationen und entsprechenden Interventionen dargestellt.

In Tabelle 1 ist eine Auswahl der häufiger verwendeten Heilpflanzen mit ihren Indikationen, so wie am Krankenhaus Meran angewendet, dargestellt.

Für die Interpretation der Ergebnisse ist es wichtig zu berücksichtigen, dass die Studie nur erhoben hat, ob ein zusätzliches komplementärmedizinisches Angebot, so wie am Krankenhaus Meran angeboten, einen Nutzen für Patientinnen mit 
Tab. 1. Häufiger verwendete Heilpflanzen am Krankenhaus Meran und ihre Indikationen

\begin{tabular}{|c|c|c|c|}
\hline Pflanze & Indikation & $\begin{array}{l}\text { Vorsichtsmaßnahmen/ } \\
\text { Kontraindikationen }\end{array}$ & $\begin{array}{l}\text { Referenzen } \\
\text { zur Evidenz }\end{array}$ \\
\hline Capsaicin & Neuropathischer Schmerz, lokale Schmerzen & $\begin{array}{l}\text { Stark durchblutungsfördernde und reizende } \\
\text { Wirkung: deshalb nicht auf Schleimhäute und } \\
\text { offene Wunden auftragen. }\end{array}$ & {$[4,5]$} \\
\hline Papain und Bromelain & $\begin{array}{l}\text { Verbesserung der Lebensqualität bei Radiotherapie } \\
\text { und Chemotherapie, Besserung der Übelkeit, } \\
\text { Schleimhautentzündungen, arthrotische Gelenksbe- } \\
\text { schwerden, schnellere Abheilung bei Paravasaten, } \\
\text { Lymphödeme }\end{array}$ & leicht gerinnungshemmende Eigenschaft & [6-8] \\
\hline $\begin{array}{l}\text { Ginseng - } \\
\text { Panax ginseng }\end{array}$ & $\begin{array}{l}\text { Steigerung der Leistungsfähigkeit und } \\
\text { Konzentration } \\
\text { Verbesserung des Allgemeinzustandes } \\
\text { Verbesserung der Müdigkeit } \\
\text { Depressive Verstimmung }\end{array}$ & $\begin{array}{l}\text { Patientinnen mit Hormonrezeptor-positiven } \\
\text { Mammakarzinomen (Ginseng hat östrogenartige } \\
\text { Aktivität) }\end{array}$ & {$[9,10]$} \\
\hline Calendula officinalis & Durch Strahlentherapie induziertes Erythem & & [11] \\
\hline Honig & $\begin{array}{l}\text { Radiogene Mukositis im Mundbereich } \\
\text { Chemotherapie-induzierte Mucositis }\end{array}$ & & [12-14] \\
\hline $\begin{array}{l}\text { Ingwer - Zingiber } \\
\text { officinalis }\end{array}$ & $\begin{array}{l}\text { Übelkeit und Erbrechen während der } \\
\text { Chemotherapie }\end{array}$ & $\begin{array}{l}\text { Wechselwirkungen mit Antikoagulantien, } \\
\text { Antihypertensiva und Antidiabetika }\end{array}$ & {$[15,16]$} \\
\hline
\end{tabular}

\section{Box 2:}

FATIGUE: Mistel (z.B. Iscador), Ginseng, Eleuterococcus, Oncophyt (Mischung japanischer Heilpilze), Vitango (Rosenwurz), Enzymtherapie (z.B. MCA Equinovo).

Durchfall: in erster Linie Flohsamenschalen (z.B. Pascomucil) oder Präparate aus Myrrhe, Kamille und Kaffeekohle (z.B.

Myrrhinil intest).

Hand-Fuß-Syndrom: Leinsamenbad, Uridinsalbe.

Nervenschädigungen: Capsaicinsalbe, Alfa Liponsäure, Leinöl. Schlafstörungen: Lavendelöl (z.B. Silexan), Passionsblume,

Baldrian, Melatonin.

Wechseljahresbeschwerden: Salbei und Lavendel,

Traubensilberkerze (z.B. Remifemin).

Ödeme: Boswellia (intrazerebral), Enzyme.

Schleimhautirritationen: Ananaswürfel, Spülungen mit Kamille, Ringelblume, Salbei, Gel aus Teebaumöl (Tebodont), Honig, Fruchtfleischöl aus Sanddorn.

Verstopfung: getrocknete Früchte, Aloe-Saft, VeraVis, Leinsamenschalen.

Übelkeit und Erbrechen: Ingwerwurzel, Pfefferminzöl, Rosenöl, Bitterpflanzen Kombinationspräparat: (z.B. Hepacur, lberogast).

Mamma-Karzinom hat, und nicht, ob einzelne angewendete Verfahren wirksam sind.

\section{Literatur}

1 Witt CM: Komplementärmedizin: Weitere Forschung ist die Basis für Integration in die Versorgung. Dtsch Arztebl 2009;1056:A1786-A1789.

2 Helfand M, Tunis S, Whitlock EP, Pauker SG, Basu A, Chilingerian J, et al: A CTSA agenda to advance methods for comparative effectiveness research. Clin Transl Sci 2011;4:188-198.
3 Institute of Medicine: What is Comparative Effectiveness Research? Initial National Priorities for Comparative Effectiveness Research. Washington D.C., The National Academies Press, 2009, p 29.

4 Mason L, Moore RA, Derry S, Edwards JE, McQuay HJ: Systematic review of topical capsaicin for the treatment of chronic pain. BMJ 2004;328:991.

5 Low PA, Opfer-Gehrking TL, Dyck PJ, Litchy WJ, O'Brien PC: Double-blind, placebo-controlled study of the application of capsaicin cream in chronic distal painful polyneuropathy. Pain 1995;62:163-168.

6 May C, Smola M, Ruda C, Scharnagl E: Randomized open controlled clinical study on the efficacy and tolerance of an oral enzyme preparation in lymphadenectomy patients. Intern J Immunother 2001;17:149-152.

7 Adamek J, Prausova J, Wald M: Enzyme therapy in the treatment of lymphedema in the arm after breast carcinoma surgery. Rozhl Chir 1997;76:203-204.

8 Parikh PM, Pai VP, Ranjan S, Swami A, Chuchelkar V, Agarwal P, et al: Phlogenzym is safe and effective in reducing morbidity of vesicant chemotherapy extravasation A prospective study. Intern J Immunother 2001;17:163-170.

9 Kim JH, Park CY, Lee SJ: Effects of sun ginseng on subjective quality of life in cancer patients: a double-blind, placebo-controlled pilot trial. J Clin Pharm Ther 2006:31:331-334.

10 Barton DL, Soori GS, Bauer BA, Sloan JA, Johnson PA, Figueras C, et al: Pilot study of Panax quinquefolius (American ginseng) to improve cancer-related fatigue: a randomized, double-blind, dose-finding evaluation: NCCTG trial N03CA. Support Care Cancer 2010;18:179-187.

11 Pommier P, Gomez F, Sunyach MP, D'Hombres A, Carrie C, Montbarbon X: Phase III randomized trial of Calendula officinalis compared with trolamine for the prevention of acute dermatitis during irradiation for breast cancer. J Clin Oncol 2004;22:1447-1453.

12 Biswal BM, Zakaria A, Ahmad NM: Topical application of honey in the management of radiation mucositis: a preliminary study. Support Care Cancer 2003;11:242-248.

13 Motallebnejad M, Akram S, Moghadamnia A, Moulana Z, Omidi S: The effect of topical application of pure honey on radiation-induced mucositis: a randomized clinical trial. J Contemp Dent Pract 2008;9:40-47.

14 Worthington HV, Clarkson JE, Eden OB: Interventions for preventing oral mucositis for patients with cancer receiving treatment. Cochrane Database Syst Rev 2006;19:CD000978.

15 Manusirivithaya S, Sripramote M, Tangjitgamol S, Sheanakul C, Leelahakorn S, Thavaramara T, et al: Antiemetic effect of ginger in gynecologic oncology patients receiving cisplatin. Int J Gynecol Cancer 2004;14:1063-1069.

16 Zick SM, Ruffin MT, Lee J, Normolle DP, Siden R, Alrawi S, et al: Phase II trial of encapsulated ginger as a treatment for chemotherapy-induced nausea and vomiting. Support Care Cancer 2009;17:563-572. 


\section{Betrachtungen zur Altershaut mit Fokus auf die Phytotherapie}

\section{Autor: Oliver Das}

Die Altershaut zeichnet sich durch diverse morphologische Veränderungen aus, welche dann auch die Neigung zu bestimmten Hauterkrankungen oder krankhaften Zuständen speziell im Alter fördern können.

Schon lange bevor Chinesen, Inder, Ägypter oder Inkas Heilpflanzen zum Zweck der Behandlung kultivierten, war die externe Anwendung von Pflanzenblättern auf der Haut als heilungsfördernde Verbände sicherlich eine der ersten und unmittelbarsten Phytotherapien überhaupt.

Wir können zwischen extrinsischer, umweltbedingter und intrinsischer, physiologischer Hautalterung unterscheiden.

Zur ersteren gehören vor allem UV-Exposition, Umweltschadstoffe, Rauchen, aber auch ungesunde Ernährung und Schlafmangel; es kommt zu lederartig gegerbt erscheinender Haut mit tiefen Falten, gelblichen Verfärbungen, UnregelmäBigkeiten sowie aktivischen Präkanzerosen.
Zur zweiten sind die nachlassende Zellregenerationsfähigkeit, verminderte Talg- und Schweißdrüsenaktivität sowie die verringerte Produktion von Antioxidantien und Östrogen zu zählen. Diese führt zu einer verdünnten, fein gefälteten Haut mit erhöhter Verletzbarkeit und deutlich verminderter Elastizität.

Histologisch spiegeln sich diese Veränderungen als Atrophie der Epidermis, Rückgang der Kollagenfasern und elastischen Fasern der Dermis und als Abnahme des Fettgewebes der Subcutis wieder.

Diesen Besonderheiten sollten bei der Behandlung der Altershaut Rechnung getragen werden.

Grundsätzlich sind die pharmakologischen Prozesse an der Haut - unabhängig davon, ob es sich um ein Phytotherapeutikum oder einen Wirkstoff anderen Ursprungs handelt - von der Resorptions- und Penetrationsfähigkeit des Vehikels abhängig.

Ausschließliche Alters-Phytotherapeutika gibt es eigentlich nicht. Die Auswahl richtet sich nach dem Krankheitsbild.

Zahlreiche Einzelsubstanzen von Pflanzen wie Alkaloide, Flavonoide, Steroide, Tannine oder ätherische Öle wie auch Pflanzeninhaltsstoffe als Gesamtextrakte kommen zur An-

Tab. 1. Häufige Symptome bzw. Hauterkrankungen im Alter und die wichtigsten dazugehörigen Phytotherapeutika

\begin{tabular}{|c|c|c|c|}
\hline Indikationen & Bemerkung & Pflanzen & Arzneimittel \\
\hline Ekzemerkrankungen & $\rightarrow$ auch Pruritus & $\begin{array}{l}\text { Hamamelis; Aloe; Bittersüßstengel (Dulca- } \\
\text { mara; Kamille; Eichenrinde; Stiefmütter- } \\
\text { chen (auch Tee); Haferstroh; Walnussblät- } \\
\text { ter (auch Tee) }\end{array}$ & $\begin{array}{l}\text { Deskin }{ }^{\circledast} \text { Lipo-Lotion, } \\
\text { Hametum }{ }^{\circledR} \text { S.; Rp; Cefabene }{ }^{\circledR} \text { S.; } \\
\text { Robugen }{ }^{\circledR} \text { Kamillensalbe, Kamillosan }{ }^{\circledR} \text { S; } \\
\text { Hewekzem Novo; andere: Rp }\end{array}$ \\
\hline Foetor ex ore & $\begin{array}{l}\text { - topisch } \\
\text { - p.o. }\end{array}$ & $\begin{array}{l}\text { - Salbei, Myrrhe, Kamille } \\
\text { - Salbei; Thymian; Anis; Wermut }\end{array}$ & Salbei Lutschpastillen, sonst Tee-Rp \\
\hline Hyperhidrosis & $\begin{array}{l}\text { - topisch } \\
\text { - oral }\end{array}$ & $\begin{array}{l}\text { - Salbei; Eichenrinde; Walnussblätter } \\
\text { - Salbei }\end{array}$ & $\begin{array}{l}\text { - Rp } \\
\text { - Sweatosan }{ }^{\circledR} \text { N Drg. }\end{array}$ \\
\hline Juckreiz & $\begin{array}{l}\text { - topisch } \\
\text { - intern } \\
\rightarrow \text { auch Ekzeme }\end{array}$ & $\begin{array}{l}\text { - Capsaicin; Pfefferminze (Menthol); } \\
\text { Thymian (thymol); Kampfer; Lavendel; } \\
\text { Weizenkleie; Haferstroh } \\
\text { - Stiefmütterchen; Sarsaparille }\end{array}$ & $\begin{array}{l}\text { - Dolenon }{ }^{\circledR} \text { Liniment S., Rp; Pruricalm } \\
\text { Lsg.; Retterspitz Gelee; Lavendel Bäder; } \\
\text { Silvaprin }{ }^{\circledR} \text { Weizen-Kleie-Extrakt; } \\
\text { sonst Rp } \\
\text { - Rp (Tee) }\end{array}$ \\
\hline Prurigo simplex & topisch & Capsaicin; Menthol; Kampher & Dolenon ${ }^{\circledR}$ Liniment S., Rp; andere: Rp \\
\hline Rosazea & topisch & $\begin{array}{l}\text { Kamille; Eichenrinde, Stiefmütterchen; } \\
\text { Haferstroh }\end{array}$ & div. Kamillenpräparate; andere: Rp \\
\hline Schmerzen & $\begin{array}{l}\text { nur adjuvant } \\
\text { - topisch } \\
\text { - systemisch }\end{array}$ & $\begin{array}{l}\text { - Capsaicin (Neuralgien), Arnika } \\
\text { (stumpfe Traumen } \\
\text { - Zitterpappel, Goldrute, Eichenrinde }\end{array}$ & $\begin{array}{l}\text { - Dolenon }{ }^{\circledR} \text { Liniment S.; Arinka Kneipp }{ }^{\circledR} \text { S., } \\
\text { Tkt., Gel } \\
\text { - Phytodolor }{ }^{\circledR} \text { Tkt. }\end{array}$ \\
\hline $\begin{array}{l}\text { Seborrhoisches } \\
\text { Ekzem }\end{array}$ & topisch & Hamamelis, Haferstroh & Deskin ${ }^{\circledR}$ Lipo-Lotion; Rp. \\
\hline Trockene Haut & $\begin{array}{l}\text { - topisch } \\
\text { - systemisch }\end{array}$ & $\begin{array}{l}\text { - Sojaöl } \\
\text { - Nachtkerzensamen-Borretschöl } \\
\text { (topisch und systemisch) }\end{array}$ & $\begin{array}{l}\text { - Balneum Hermal Ölbad; Linola } \\
\text { Gamma S., Eucerin Omega Cr. } \\
\text { - Quintesal Kps., Epogam Kps. }\end{array}$ \\
\hline Wundheilung & - systemisch & $\begin{array}{l}\text { Kamille; Ringelblume; Arnika; Johannis- } \\
\text { kraut; Sonnenhut (Echinacea); Eichenrin- } \\
\text { de; Beinwell; Aloe; Zaubernuss (Hamame- } \\
\text { lis); Spitzwegerich; Walnussblätter } \\
\text { - Ananas- und Papaya-Enzyme }\end{array}$ & $\begin{array}{l}\text { - Robugen }{ }^{\circledR} \text { Kamillensalbe, Kamillosan }{ }^{\circledR} \text { S; } \\
\text { Hewekzem Novo; Calendumed S.; Arnika } \\
\text { Kneipp }{ }^{\circledR} \text { Tkt., S.; andere Rp } \\
\text { - Phlogenzym }{ }^{\circledR} \text { Drg., Bromelain POS }{ }^{\circledR} \text {, } \\
\text { Wobe-Mugos }{ }^{\circledR} \text { Tbl. }\end{array}$ \\
\hline
\end{tabular}


wendung. Bei Letzteren hofft man auf synergistische Wirkungen bei gleichzeitig geringerer Nebenwirkungsrate.

Jüngere Studien, insbesondere zu einem klar definierten Thema wie Phythotherapeutika und Altershaut, gibt es kaum.

Lediglich 2 spezifische Studien der letzten 10 Jahre lassen sich hierzu anführen:

1.) «Externe Anwendung von Weihrauch-Extrakt bei lichtgeschädigter <Altershaut - Ergebnisse einer Pilotstudie mit 15 Patientinnen», durchgeführt an der Dermatologischen Abteilung der Universitätsklinik Brescia von Pedretti et al. Bei dieser doppelblinden, randomisierten Studie wurde jeweils an einer Gesichtshälfte der Patientinnen mit lichtgeschädigter Altershaut eine Creme mit $0,5 \%$ eines Weihrauch-Extraktes angewandt. Die Studie zeigte mehrheitlich günstigere Ergebnisse auf Seiten der mit dem Boswellia-Extrakt behandelten Gesichtshälften und erschien in Deutschland 2010 in der Zeitschrift für Phytotherapie [1].

2.) Die zweite Studie «Hamamelis-Salbe in der Pflege der trockenen Altershaut» wurde an der Dermatologischen Klinik in Augsburg durchgeführt und erschien 2005 in der Zeitschrift für Physiotherapie [2]. In der Studie, an der 89 Patienten teilnahmen, konnte die Wirksamkeit von Hamamelis-Salbe bei verschiedenen altersbedingten Hautbeschwerden gezeigt werden.

Weitere Studien gibt es zu diversen Erkrankungszuständen der Haut oder zu Wundbehandlungen, welche sicherlich ebenfalls für die Altershaut eine Rolle spielen, aber nicht nur die Altershaut betreffen.

Im letzten Teil des Vortrages gehe ich im Einzelnen auf häufige Symptome bzw. Hauterkrankungen im Alter und die wichtigsten dazugehörigen Phytotherapeutika ein.

Dies ist in Tabelle 1, welche ich auszugsweise aus dem Lehrbuch «Phytotherapie bei Hauterkrankungen» [Augustin M Hoch Y: Phytotherapie bei Hauterkrankungen. Urban \& Fischer, 2004, S. 267-271] entnommen habe und das ein umfassendes und gutes Standardwerk für den dermatologischen Bereich ist, dargestellt.

\section{Literatur}

1 Schulz V: Externe Anwendung von Weihrauch-Extrakt bei lichtgeschädigter «Altershaut»- Ergebnisse einer Pilotstudie mit 15 Patientinnen. Z Phytother 2010;31:201-202.

2 Welzel J, Walther C, Kieser M, Wolff HH: Hamamelis-Salbe in der Pflege der trockenen Altershaut. Z Phytother 2005;26:6-13.

\section{Arzneimittelinteraktionen bei multimorbiden Patienten - welche Rolle spielen pflanzliche Arzneimittel?}

\section{Autor: Matthias Unger}

Ältere Menschen sind häufig multimorbide, d.h., sie müssen täglich mehrere Arzneimittel einnehmen, was die Wahrscheinlichkeit von Arzneimittelinteraktionen stark erhöht. Durch die zusätzliche Einnahme pflanzlicher Arzneimittel steigt das Risiko für Interaktionen weiter an. In diesem Referat sollen die den Arzneimittelinteraktionen zugrunde liegenden Prinzipien erläutert und die Wahrscheinlichkeit klinisch relevanter Wechselwirkungen zwischen pflanzlichen und synthetischen Arzneimitteln bewertet werden.

Die Bioverfügbarkeit von synthetischen Arzneimitteln wird durch zwei grundlegende Mechanismen beeinflusst: metabolische Veränderungen durch Enzymsysteme sowie Transportvorgänge durch einwärts- bzw. auswärtsgerichtete Influx- oder Efflux-Pumpen. Beide Mechanismen können durch Arzneistoffe und sekundäre Pflanzeninhaltsstoffe sowohl gehemmt als auch induziert werden. Während die metabolischen Veränderungen vorwiegend durch Cytochrom-P450 (CYP)-Enzyme (Phase-I-Metabolismus) sowie Glucuronosyltransferasen (Phase-II-Metabolismus) verursacht werden, sind bei den Transportern hauptsächlich der Effluxtransporter P-Glykoprotein für den auswärtsgerichteten Transport und die OATPs (Organic Anion Transporting Polypeptides) für den einwärtsgerichteten Transport von Molekülen verantwortlich. Die häufigsten und wichtigsten Arzneimittelinteraktionen treten auf, wenn die Bioverfügbarkeit von Arzneistoffen durch eine Inhibition oder Induktion des Arzneistoffmetabolismus oder der Arzneistoffabsorption bzw. -elimination erhöht oder vermindert wird. Solche Wechselwirkungen werden als pharmakokinetische Interaktionen bezeichnet. Bei pflanzlichen Arzneimitteln sind in den vergangenen Jahren klinisch relevante pharmakokinetische Interaktionen beschrieben worden, wobei berücksichtigt werden muss, dass die Komplexität der verwendeten Pflanzenextrakte sowie fehlende Angaben zu ihrer Zusammensetzung eine Interpretation und schlüssige Bewertung der Studienergebnisse in vielen Fällen erheblich erschweren.

Hyperforinhaltige Johanniskrautextrakte führen nach einigen Tagen vor allem in Leber und Dünndarm zu einer gesteigerten Expression (Induktion) von CYP-Enzymen und P-Glykoprotein. Dadurch wird die Bioverfügbarkeit von zahlreichen CYP- und/oder P-Glykoprotein-Substraten klinisch relevant erniedrigt. Zum Beispiel nimmt die AUC von Simvastatin, dessen First-Pass-Effekt hauptsächlich durch die intestinale CYP3A4-Expression bedingt ist, nach der Einnahme eines hyperforinhaltigen Hypericumextraktes um $62 \%$ ab (Tab. 1) [1]. Im Gegensatz dazu konnte eine klinisch relevante Inhibition des Arzneistoffmetabolismus oder -transports durch die in Europa zugelassenen pflanzlichen Arzneimittel bisher nicht zweifelsfrei belegt werden (Tab. 1). Die bei In-vivo-Studien 
Tab. 1. Ausgewählte Interaktionsstudien mit Phytopharmaka, die besonders häufig von älteren Patienten eingenommen werden

\begin{tabular}{|c|c|c|c|c|c|c|}
\hline Extrakt & $\begin{array}{l}\text { Dosis } \\
\mathrm{mg} / \mathrm{Tag}\end{array}$ & $\begin{array}{l}\text { Dauer } \\
\text { (Tage) }\end{array}$ & $\mathrm{N}$ & $\begin{array}{l}\text { Komedika- } \\
\text { tion }\end{array}$ & Effekt & Lit. \\
\hline Hypericum & 900 & 14 & 16 & Simvastatin & AUC $\downarrow$ \ $(62 \%)$ & [1] \\
\hline Crataegus & 900 & 21 & 8 & Digoxin & AUC \(7\%) & [2] \\
\hline Valeriana & 375 & 28 & 12 & $\begin{array}{l}\text { Midazolam } \\
\text { Koffein } \\
\text { Debrisoquin }\end{array}$ & $\begin{array}{l}\text { CYP3A } 4 \downarrow t \\
\text { CYP1A2 } \downarrow \uparrow \\
\text { CYP2D6 } \downarrow \uparrow\end{array}$ & {$[3]$} \\
\hline Silybum & 600 & 12 & 6 & $\begin{array}{l}\text { Irinotecan } \\
\text { SN-38 }\end{array}$ & $\begin{array}{l}\text { AUC } ₫(14 \%)^{*} \\
\mathrm{AUC} \downarrow(5 \%)^{*}\end{array}$ & [4] \\
\hline Silybum & 540 & 14 & 6 & $\begin{array}{l}\text { Losartan } \\
\text { EXP-3174 }\end{array}$ & $\begin{array}{l}\text { AUC } \uparrow(108 \%) \\
\text { AUC } \downarrow(15 \%)\end{array}$ & {$[5]$} \\
\hline Ginkgo & 240 & 12 & 14 & Voriconazol & $\mathrm{AUC} \downarrow(17 \%)^{*}$ & [6] \\
\hline \multicolumn{7}{|c|}{$\begin{array}{l}\text { N: Probandenzahl; AUC: Area under the curve (Fläche unter der } \\
\text { Blutspiegelkurve); } \\
\text { *: statistisch nicht signifikant. }\end{array}$} \\
\hline
\end{tabular}

beobachteten Änderungen der Arzneistoff-Bioverfügbarkeit durch z.B. Weißdorn- und Baldrianpräparate sind klinisch nicht relevant $[2,3]$.

Tankanow et al. [2] untersuchten den Einfluss eines Spezialextraktes aus den getrockneten Blättern und Blüten von $\mathrm{Cra}$ taegus monogyna und C. oxyacantha $(900 \mathrm{mg} / \mathrm{d})$ auf die Bioverfügbarkeit des P-Glykoprotein-Substrates Digoxin über einen Zeitraum von 21 Tagen bei 8 gesunden Probanden. Der Weißdorn-Spezialextrakt führte nicht zu einer klinisch signifikanten Veränderung der maximalen Plasmakonzentration, Eliminationshalbwertszeit oder AUC von Digoxin, so dass die gleichzeitige Einnahme von Weißdornpräparaten mit Arzneistoffen, deren Bioverfügbarkeit überwiegend durch die Aktivität von P-Glykoprotein bestimmt wird, mit hoher Wahrscheinlichkeit nicht zu Arzneimittelinteraktionen führt.

In einer 2005 von Gurley et al. [3] veröffentlichten In-vivo-Phenotyping-Studie wurde der Einfluss eines Baldrian-Präparates auf die Aktivität von CYP3A4, CYP1A2 und CYP2D6 untersucht [3]. Bei 12 gesunden Probanden zeigte die Applikation von täglich $375 \mathrm{mg}$ Baldrianextrakt nach einem Zeitraum von 28 Tagen keine signifikante Veränderung der CYP-Enzym-Aktivität (Tab. 1) [3], so dass eine klinisch relevante Beeinflussung der Pharmakokinetik von Substraten der CYP-Enzyme 1A2, 2D6 und 3A4 durch Baldrianpräparate nicht zu erwarten ist.

Die bisher für Mariendistelextrakte( $>70 \%$ Silymarin) veröffentlichten In-vitro- und In-vivo-Studien weisen auf eine Beeinflussung des Arzneistoffmetabolismus durch Silymarin hin. Da in vitro eine zeitabhängige irreversible Inaktivierung von CYP-Enzymen durch Silymarin gezeigt werden konnte [4, 7], erscheint eine klinisch relevante Beeinflussung der Arzneistoffbioverfügbarkeit durch Mariendistelextrakte zunächst plausibel [7, 8]. Van Erp et al. [4] untersuchten 2005 den Einfluss einer Zubereitung aus Mariendistelfrüchten auf die Pharmakokinetik von Irinotecan, das in vivo durch Carboxyesterasen in das zytotoxische SN-38 umgewandelt wird. Die pharmakokinetischen Parameter (z.B. AUC) von Irinotecan, SN-38 und SN-38-Glucuronid waren nach der 4- bzw. 12-tägigen Einnahme des Mariendistelpräparates nahezu unverändert (Tab. 1) [4]. Han et al. untersuchten 2009 den Einfluss von $540 \mathrm{mg}$ Mariendistelextrakt auf die Bioverfügbarkeit von Losartan und seinem durch CYP2C9 und CYP3A4 gebildeten aktiven Metaboliten EXP-3174 über einen Zeitraum von 14 Tagen bei sechs Patienten. Da sich die AUC von Losartan statistisch signifikant um 108 \% erhöhte und die AUC von EXP3174 um 15\% abnahm (Tab. 1), könnte die in vitro beobachtete starke und irreversible Inaktivierung von CYP2C9 und CYP3A4 durch Silybin $[4,5,7]$ auch in vivo relevant sein. Die geringe Probandenzahl ( $\mathrm{N}=6$; Tab. 1) ist allerdings ein großes Manko dieser Studie, so dass eine abschließende Bewertung zur In-vivo-Beeinflussung der CYP2C9- und CYP3A4-Aktivität durch Mariendistelextrakte noch nicht möglich ist.

Eine klinisch relevante Beeinflussung der Bioverfügbarkeit von Arzneistoffen konnte auch bei Ginkgoextrakten nicht nachgewiesen werden [7, 9], obwohl zahlreiche In-vitro-Studien auf Interaktionen in vivo hindeuten [9]. Bei Voriconazol, das hauptsächlich über CYP2C19 und CYP3A4 metabolisiert wird, wurde bei 14 Probanden über einen Zeitraum von 12 Tagen durch die tägliche Einnahme von $240 \mathrm{mg}$ Ginkgoextrakt lediglich eine (statistisch nicht signifikante) Abnahme der Voriconazol-AUC um 17\% festgestellt (Tab. 1) [6]. Da hinsichtlich der Beeinflussung von metabolischen Vorgängen oder Transportprozessen durch Phytopharmaka nur selten eine positive In-vitro-in-vivo-Korrelation vorliegt, sollten Ergebnisse aus In-vitro-Studien vorsichtig interpretiert werden [7-9].

\section{Literatur}

1 Sugimoto K, Ohmori M, Tsuruoka S, Nishiki K, Kawaguchi A, Harada K, Arakawa M, Sakamoto K, Masada M, Miyamori I, Fujimura A: Different effects of St John's wort on the pharmacokinetics of simvastatin and pravastatin. Clin Pharmacol Ther 2001;70:518-524.

2 Tankanow R, Tamer HR, Streetman DS, Smith SG, Welton JL, Annesley T, Aaronson $\mathrm{KD}$, Bleske BE: Interaction study between digoxin and a preparation of hawthorn (Crataegus oxyacantha). J Clin Pharmacol 2003;43:637-642.

3 Gurley BJ, Gardner SF, Hubbard MA, Williams DK, Gentry WB, Khan IA, Shah A: In vivo effects of goldenseal, kava kava, black cohosh, and valerian on human cytochrome P450 1A2, 2D6, 2E1, and 3A4/5 phenotypes. Clin Pharmacol Ther 2005;77:415-426.

4 Van Erp NP, Baker SD, Zhao M, Rudek MA, Guchelaar HJ, Nortier JW, Sparreboom A, Gelderblom H: Effect of milk thistle (Silybum marianum) on the pharmacokinetics of irinotecan. Clin Cancer Res 2005;11:7800-7806.

5 Han Y, Guo D, Chen Y, Chen Y, Tan ZR, Zhou HH: Effect of silymarin on the pharmacokinetics of losartan and its active metabolite E-3174 in healthy Chinese volunteers. Eur J Clin Pharmacol 2009;65:585-591.

6 Lei HP, Wang G, Wang LS, Ou-yang DS, Chen H, Li Q, Zhang W, Tan ZR, Fan L, He YJ, Zhou HH: Lack of effect of Ginkgo biloba on voriconazole pharmacokinetics in Chinese volunteers identified as CYP2C19 poor and extensive metabolizers. Ann Pharmacother 2009;43:726-731.

7 Unger M: Pharmacokinetic drug interactions by herbal drugs: critical evaluation and clinical relevance. Wien Med Wochenschr 2010;160:571-577.

8 Unger M: Pharmacokinetic drug interactions between anticancer therapeutics and drugs of complementary medicine: mechanisms and clinical relevance. Forsch Komplementmed 2011;18:213-218.

9 Unger M: Pharmacokinetic drug interactions involving Ginkgo biloba. Drug Metab Rev 2013;45:353-385. 


\section{Disclosure Statement}

Reinhard Saller: Es besteht kein Interessenkonflikt.

Siegfried Kasper: Prof. Kasper erhielt Honorare für Beraterverträge und Vorträge von Angelini, AstraZeneca, Bristol-Myers Squibb, Eli Lilly, GlaxoSmithKline, Janssen, Lundbeck, Merck Sharp and Dome (MSD), Neuraxpharm, Novartis, Organon, Pierre Fabre, Pfizer, Schwabe Sepracor, und Servier und Forschungsunterstützung von AstraZeneca, Bristol Myers-Squibb, Eli Lilly, GlaxoSmithKline, Lundbeck, Organon, Sepracor und Servier.

Wilfried Dimpfel: Es besteht kein Interessenkonflikt.

Thomas Pfister: Es besteht kein Interessenkonflikt.

Matthias Rostock: Der Autor gibt an, dass keine Interessenkonflikte bestehen.
Claudia Witt: Die von Claudia Witt geleitete Studie wurde von der Landesregierung Südtirol gefördert.

Christian Thuile: Der Autor erklärt, dass kein Conflict of Interest besteht.

Oliver Das: Oliver A.C. Das hat bei der Erstellung seines Vortrages bis auf Unterstützungen bei der Literaturrecherche durch die Firmen Schwabe Pharma AG und Phytoceuticals Ltd keine weitere Hilfe durch Dritte erhalten.

Matthias Unger: Es besteht kein Interessenkonflikt. 